Purdue University Purdue e-Pubs

2014

\title{
3D Reconstruction and Design of Porous Media from Thin Sections
}

\author{
K. K. Bodla \\ Purdue University \\ S. V. Garimella \\ Purdue University, sureshg@purdue.edu
}

Follow this and additional works at: http://docs.lib.purdue.edu/coolingpubs

Bodla, K. K. and Garimella, S. V., "3D Reconstruction and Design of Porous Media from Thin Sections" (2014). CTRC Research Publications. Paper 220.

http://dx.doi.org/http://dx.doi.org/10.1016/j.ijheatmasstransfer.2014.02.006

This document has been made available through Purdue e-Pubs, a service of the Purdue University Libraries. Please contact epubs@purdue.edu for additional information. 


\title{
3D Reconstruction and Design of Porous Media from Thin Sections ${ }^{1}$
}

\author{
Karthik K. Bodla ${ }^{a}$, Suresh V. Garimella ${ }^{a}{ }^{2}$, and Jayathi Y. Murthy ${ }^{\mathrm{b}}$ \\ ${ }^{a}$ School of Mechanical Engineering and Birck Nanotechnology Center \\ Purdue University, 585 Purdue Mall, West Lafayette, IN 47907 USA \\ ${ }^{b}$ Department of Mechanical Engineering \\ University of Texas at Austin, 1 University Station C2200, Austin, TX 78712-0292 USA
}

\begin{abstract}
Characterization and design of fluid-thermal transport through random porous sintered beds is critical for improving the performance of two-phase heat transport devices such as heat pipes. Two-dimensional imaging techniques are quite well developed and commonly employed for microstructure and material characterization. In this study, we employ 2D image data (thin sections) for measuring critical microstructural features of commercial wicks for use in correlation-based prediction of transport properties. We employ a stochastic characterization methodology based on the two-point autocorrelation function, and compare the predicted properties such as particle and pore diameters and permeability with those from our previously published studies, in which 3D x-ray microtomography data was employed for reconstruction. Further, we implement a reconstruction technique for reconstructing a three-dimensional stochastic equivalent structure from the thin sections. These reconstructed domains are employed for predicting effective thermal conductivity, permeability and interfacial heat transfer coefficient in singlephase flow. The current computations are found to compare well with models and correlations from the literature, as well as our previous numerical studies. Finally, we propose a new parametrized model for the design of porous materials based on the nature of the two-point autocorrelation functions. Using this model, we reconstruct sample three-dimensional microstructures, and analyze the influence of various parameters on fluid-thermal properties of interest. With advances in additive manufacturing techniques, such an approach may eventually be employed to design intricate porous structures with properties tailored to specific applications.
\end{abstract}

\footnotetext{
${ }^{1}$ Submitted for possible publication in International Journal of Heat and Mass Transfer, August 2013

${ }^{2}$ Corresponding author: Tel. 17654945621 ; sureshg@ purdue.edu
} 


\section{Nomenclature}

$\begin{array}{llll}a, b, c, d & \text { fitting coefficients } & r & \text { radial coordinate } \\ A & \text { area } & r_{g} & \text { particle size } \\ B o & \text { Bond number } & R & \text { correlated 3D image } \\ C & \text { specific heat } & s & \text { specific surface area } \\ C_{E} & \text { Ergun's coefficient } & S_{1}, S_{2} & \text { correlation functions } \\ D_{H} & \text { hydraulic diameter } & T & \text { temperature } \\ D_{p}, D_{p, 3 D} & \text { particle diameter } & u, U & \text { velocity } \\ f(x) & \text { indicator function } & \text { Greek symbols } \\ F & \text { filter function } & \rho & \text { density } \\ h_{s f} & \text { heat transfer coefficient } & \sigma & \text { surface tension } \\ \boldsymbol{J} & \text { heat flux vector } & \theta & \text { contact angle } \\ k & \text { thermal conductivity } & \Phi & \text { porosity } \\ K & \text { permeability } & \mu & \text { viscosity } \\ M, N & \text { image dimensions } & \text { Subscripts } & \\ N u_{s f} & \text { Nusselt number } & 3 D & \text { calculated from 3D XMT data } \\ P & \text { pressure } & c a p & \text { capillary } \\ q ” & \text { heat flux } & f & \text { fluid side } \\ & & s f & \text { solid-fluid }\end{array}$

Keywords: porous media, wick structures, 3D reconstruction, effective thermal conductivity, permeability, interfacial heat transfer, autocorrelation function, designed porous media

\section{Introduction}

Heat pipes and vapor chambers are two-phase heat transport devices that offer a passive and compact means of transporting heat over long distances without a substantial drop in temperature [1,2]. A heat pipe consists of a sealed chamber containing a working fluid such as water which transports heat through phase change. It exploits the capillary action generated by the wicking material lining the inside to passively transport the working fluid. Because their effective thermal conductivity can be two to three orders of magnitude higher than for solid metal structures of the same dimensions, these devices find widespread application in thermal management [3]. Some of the specific applications of such two-phase heat transport devices in the electronics cooling industry are for hot-spot cooling and heat spreading [4].

Estimation of transport properties of the wick structures, as well as enhanced wick designs, are essential for improving the performance of heat pipes. Dullien [5] reviewed various experimental 
measurement techniques available for characterizing transport properties of porous media. Numerical modeling offers an alternative means of transport characterization. In our previous studies $([6,7])$, a thorough characterization of single-phase properties as well as evaporative performance of monoporous sintered copper wicks was performed. The limitations of present-day experimental methods and commonly used analytical expressions in characterizing heat transfer properties, such as effective thermal conductivity and capillary pressure, were demonstrated. A recent study [8] provided a detailed review of advances in the prediction of fluid-thermal transport properties of ideal and realistic porous media.

Recent advances in 3D imaging techniques, employing both destructive and non-destructive approaches, as well as a corresponding development of 3D image-based mesh generation, have led to a surge in the number of studies employing such methods for numerical characterization of transport properties of random porous materials. In our past studies $[6,9]$, an x-ray microtomography (XMT) based numerical characterization technique was developed for simulating fundamental thermal transport properties of open-cell metal foams. Subsequently, this methodology was employed for predicting the transport properties of commercial wick structures; single-phase transport properties were simulated in [6], followed by capillary and evaporative performance in [7]. Example studies in other areas include prediction of petrophysical characteristics of porous rocks [10], and micromechanical characterization of reinforced composites [11].

3D imaging techniques provide a number of obvious benefits such as accurate and detailed representation of the scanned volume, and a requirement of little to no pre-processing. However, they also suffer from a few fundamental limitations. Typically, there is a tradeoff between the scanning resolution and the size of the sample being scanned, as discussed in [9]. Along similar lines, the high present-day cost and limitations of x-rays in detecting denser materials limit the technique to a few exotic applications. Further, feature sizes in the materials of interest are on the order of a few microns, while popular nanometer-resolution techniques, such as 3D Transmission Electron Microtomography (TEMT), are typically employed to characterize the structures with characteristic lengths on the order of few nanometers [12]. 2D scanning techniques such as optical microscopy, scanning and transmission electron microscopy, and scanning probe microscopy are quite well developed [13]. 2D scanning is also rapid and inexpensive, and is employed widely. Porous media characterization techniques based on 2D images are thus desirable for a broad range of applications.

Two-dimensional scans may be used for measuring microstructural features such as porosity, specific surface area, and pore and particle sizes of the porous media. By employing a correlation-based approach, these characteristics may then be used for rapidly predicting transport properties such as permeability via the widely used Kozeny-Carman correlation [1]. In this work we adopt a statistical analysis procedure based on the two-point autocorrelation function developed by Blair et al. [14] and detailed in the 
following section. We apply this technique to individual 2D slices taken from our past 3D scans [6], and measure microstructural features. A detailed comparison of pore and particle sizes, as well as permeability from the current study and those obtained from [6], is performed.

Three-dimensional microstructure reconstruction typically requires a stack of $2 \mathrm{D}$ data, such as that obtained via X-ray microtomography. However, when the porous material of interest is homogeneous and largely isotropic, stochastically equivalent 3D microstructures, corresponding to the 2D scan image may be obtained. A number of techniques such as the Joshi-Quiblier-Adler (JQA) approach [15], the single cut-Gaussian Random Field (GRF) approach [15], and the Karhunen-Loeve expansion-based approach [16] are available for accomplishing this reconstruction. The JQA approach was originally developed by Joshi [17] for reconstruction of stochastically similar 2D data, and later extended by Quiblier [18] to three dimensions. Optimization techniques such as the simulated annealing (SA)-based reconstruction technique developed by Yeong and Torquato [19] are also commonly used. Other recently developed approaches include employment of multi-point statistics for 3D reconstruction [20]. In this work we employ a modified JQA approach, similar to the one proposed in [15], and use it to reconstruct 3D microstructures from 2D scan images. These 3D microstructures are then used for computing transport characteristics such as permeability, effective thermal conductivity and interfacial heat transfer coefficient for single-phase flow through the microstructure. Examples of other recent publications that reconstruct $3 \mathrm{D}$ porous media from stochastic information computed from $2 \mathrm{D}$ images include Jiang et al. [21], Zhang and $\mathrm{Du}$ [22], and Chen et al. [23].

In addition to simulating transport properties of existing microstructures, a novel parameterized model based on the typical functional form of the autocorrelation function for the porous material is also proposed. For this, a spring-mass-damper system analogy is employed, and the influence of various parameters on transport characteristics, viz., effective thermal conductivity, permeability and interfacial heat transfer, is analyzed. Similar approaches in the literature include models for generating "Debye random media" proposed by Debye and Bueche [24] and Debye et al. [25], and discussed in detail in Yeong and Torquato [19]. This model consists of a single parameter, the correlation length, while our model has three independent parameters as described in Section 4. Employing such a parameterized model with controllable parameters, new microstructures with tailored transport characteristics may be designed. The following section describes the autocorrelation function, and the correlation-based approach developed for a quick estimation of transport.

\section{Correlation-based analysis of transport characteristics}

\subsection{Spatial Correlation Functions}


Stochastic porous media may be analyzed by means of a variety of correlation functions. Binary porous materials such as the sintered metals considered here may be represented via an indicator function, $f(x)$, defined as follows:

$$
f(x)=\left\{\begin{array}{l}
1 \text { if } x \in \text { Pore space } \\
0 \text { if } x \in \text { Material }
\end{array}\right.
$$

Binarised digital images through any cross-section of the material represent a discretized version of this indicator function [14]. Furthermore, when the medium is isotropic, the sum of $f$ over the entire image of any cross-section provides an estimate of porosity of the material, $\Phi$, within statistical uncertainty. Formally, porosity may be defined as the one-point correlation function, $S_{l}$; in other words, given an indicator function, the porosity of a porous material is the probability of finding a randomly selected point in the pore space [14]:

$$
S_{1}=\langle f(x)\rangle=\phi
$$

Here, \langle\rangle indicates area averaging for the case of 2D data. Along similar lines, a two-point autocorrelation function, $S_{2}$, may be defined as the probability of finding two points, located at $\boldsymbol{r}_{1}$ and $\boldsymbol{r}_{2}$, such that both lie in the pore space of the material [14]:

$$
S_{2}\left(\mathbf{r}_{1}, \mathbf{r}_{2}\right)=\left\langle f\left(\mathbf{r}_{1}\right) f\left(\mathbf{r}_{2}\right)\right\rangle
$$

Again, in Eq. (3), \langle\rangle represents spatial averaging. It may be noted that when the material is isotropic, $S_{2}$ depends only on the separation distance, $r=\left|\boldsymbol{r}_{1}-\boldsymbol{r}_{2}\right|$, and not on the specific location of the individual points [14]. For such cases, $S_{2}(r)$ may be simply computed as:

$$
S_{2}(r)=\langle f(x) f(x+r)\rangle
$$

This property has significant uses. Firstly, the autocorrelation function may be computed along various directions and employed to analyze the microstructural isotropy of a material - if the autocorrelation function is the same along all directions, the material is statistically isotropic, and vice versa. Along similar lines, $S_{2}$ may be computed from a single 2D image, and employed to generate an isotropic stochastically equivalent 3D structure as described in detail in [15] and Section 3.2.

Other higher-order functions, such as generic n-point correlation functions $(n>2)$, and lineal path functions, are also typically employed for characterizing porous materials (e.g., [19] and [26]), at the cost of increasing computational effort. In this work, we restrict ourselves to the lower order, one- and twopoint correlation functions, which are the most commonly employed functions in the literature.

Several algorithms exist for computing the one- and two-point correlation functions from discretized 2D images. One such algorithm may be found in [27], and is widely employed for isotropic, 
homogeneous porous media. Using this approach, the one-point correlation function, $S_{l}$, is computed as follows:

$$
S_{1}=\phi=\frac{1}{M N} \sum_{\substack{1 \leq i \leq M \\ 1 \leq j \leq N}} f_{i, j}
$$

Here, the image size is assumed to be $M \times N$ pixels. Similarly, the autocorrelation function is computed for each pixel $(m, n)$ in the image as follows:

$$
S_{2}(m, n)=\frac{1}{i_{\max } j_{\max }} \sum_{\substack{1 \leq i \leq i_{\max } \\ 1 \leq j \leq j_{\max }}} f_{i, j} f_{i+m, j+n}
$$

In this equation, $i_{\max }=M-m$, and $j_{\max }=N-n$, respectively [27]. Once computed, the data are averaged to produce the direction-independent, one-dimensional two-point autocorrelation function, $S_{2}(r)$. For this, the first pixel in the image is considered as the origin, and autocorrelation functions along various polar directions with discrete increments are considered. For points not coinciding with the pixel centroids, bilinear interpolation is employed [27]. Figure 1 a) shows a typical computational grid with pixel centroids and actual lattice points, while Figure $1 \mathrm{~b}$ ) shows the $S_{2}$ function computed for our samples, along with the 2D images employed, taken from [6]. Further details of this approach may be found in [27].

\subsection{Microstructural Characteristics from Autocorrelation Functions}

Correlation functions may be employed to characterize the microstructure of stochastic porous materials. As noted earlier, $S_{l}$ is simply the porosity of the material of interest. Similarly, the two-point autocorrelation function has several interesting characteristics. For all isotropic materials without longrange order, the following properties may be readily derived:

$$
S_{2}(r=0)=\phi ; \lim _{r \rightarrow \infty} S_{2}(r)=\phi^{2}
$$

Further, Debye et al. [25] showed that the surface area per unit volume or the specific surface area $s$ of the porous material may be readily obtained from the slope of the two-point autocorrelation function. Employing a 2D image, such as the one in the current study, the surface area per unit volume may be computed as follows [19]:

$$
s=-\left.\pi \frac{d}{d r}\left(S_{2}(r)\right)\right|_{r=0}
$$

Specific surface area is useful in estimating the effective pore diameter of porous media. As noted in Dullien [5], the effective pore diameter of any porous material may be defined as follows: 


$$
D_{H}=\frac{4 \phi}{s}
$$

Blair et al. employed a two-point autocorrelation function for estimating permeability of sandstones, via the Kozeny-Carman correlation [1]. By considering ideal, non-penetrable and penetrable sphere models of packed beds, they demonstrated the utility of the autocorrelation function in computing the particle size in addition to the specific surface area $s$. It was shown that when the autocorrelation function showed a distinct minimum, dropping below the asymptotic value of $\Phi^{2}$ (Eq. (7)), the strong negative correlation implied the presence of particles of nearly uniform size [14]. Further, the oscillatory behavior of $S_{2}$ around the asymptotic value, observed for certain cases, was attributed to the roundness of particles. It was demonstrated that rounder particles showed significant oscillations, while angular ones, such as the sandstone microstructures, corresponded to non-oscillatory behavior in the computed autocorrelation function. Furthermore, the location of the first minimum (in cases with oscillatory behavior), and the location at which the autocorrelation function became asymptotic (in cases with non-oscillatory behavior), $r_{g}$ (Figure $1 \mathrm{~b}$ )), was correlated to the average particle size as follows:

$$
D_{p}=\left\{\begin{array}{l}
\frac{2 r_{g}}{1.3}, \text { for } S_{2} \text { with distinct minimum } \\
r_{g}, \text { for } S_{2} \text { with no distinct minimum }
\end{array}\right.
$$

The pore and particle diameters thus estimated may be employed for predicting transport characteristics. Permeability is typically estimated via the Kozeny-Carman correlation [1]:

$$
K=\frac{D_{p}^{2} \phi^{3}}{150(1-\phi)^{2}}
$$

Similarly, hydraulic diameter may be used to gain a first estimate of capillary pressure employing the Young-Laplace equation [7]:

$$
P_{\text {cap }}=\frac{4 \sigma \cos \theta}{D_{H}}
$$

Here, $\sigma$ and $\theta$ are the surface tension and the contact angle of the liquid, respectively.

In this study, we have employed the two-point autocorrelation function for estimating the microstructural characteristics for three sintered copper wick microstructures fabricated at a porosity of approximately 61-63\%, and consisting of particles in the size ranges, $45-75 \mu \mathrm{m}, 106-150 \mu \mathrm{m}$, and 250$355 \mu \mathrm{m}$, respectively. It may be noted that the original scan resolution employed in [6] was $5.5 \mu \mathrm{m}$, and based on the physical dimension of the volumes scanned, we have access to about $900 \times 1300 \times 100$, $750 \times 1300 \times 100$, and $700 \times 1300 \times 100$ pixels of data for the small, intermediate and large-sized particle samples, respectively. In this study, we employ $2 \mathrm{D}$ images of size $350 \times 350$ pixels for computing the 
stochastic functions described. For the small and intermediate particle sizes, the image resolution employed was $5.5 \mu \mathrm{m}$, while for the largest particle-size sample, a $11 \mu \mathrm{m}$ resolution was used so as to include a sufficient number of particles in the $350 \times 350$ pixel image. Five images spanning the entire 3D space are employed per sample to average out the randomness in the sample. A detailed comparison of our present results, along with those from our past study [6], is provided in Section 6.1.

\section{Porous medium reconstruction}

\subsection{Reconstruction from Image Stack}

The correlation-based analysis presented in the previous section provides a quick means of predicting transport properties of porous materials. Such methods may be employed to analyze large sets of samples. However, when a detailed analysis or high levels of accuracy are desirable, a suitable alternative technique is the use of $3 \mathrm{D}$ computational volumes for numerically estimating fluid-thermal transport properties. This section briefly describes the various 3D reconstruction methods available in the literature, along with the method employed in the current study which is based on the modified JQA approach, proposed in [28].

Accurate 3D representations of random porous materials may be obtained via destructive or nondestructive 3D imaging techniques, each with its own merits and drawbacks. Examples of the former include Focused Ion Beam (FIB)-based milling followed by 2D SEM imaging, FIB-SEM [29], to produce a series of $2 \mathrm{D}$ scans that may be subsequently employed to reconstruct the $3 \mathrm{D}$ volume of interest. Similarly, non-destructive techniques such as x-ray microtomography may be employed to produce 2D scan data in a non-intrusive fashion. In our previous studies ([6], [9] and [30]) we have employed such a technique for reconstructing random aluminum foams and sintered copper microstructures. The reconstructed volumes were employed to compute fundamental single- and two-phase transport characteristics.

Employing destructive techniques, high resolutions are realizable. However, being destructive, they have certain inherent limitations such as the inability to accurately image all the pore space. Further, these techniques often require a significant amount of processing owing to the underlying milling process involved, and typically, the higher the desired resolution, the longer is the required processing time. On the other hand, $\mathrm{x}$-ray microtomography techniques are quick, and require little to no processing prior to scanning. However, x-rays have limited penetrability, and the penetrability decreases with increase in the density of the material being imaged. Therefore, rapid reconstruction techniques based on 2D scan images are desirable.

\subsection{Reconstruction from Thin Sections}




\subsubsection{Modified Joshi-Quiblier-Adler (JQA) approach}

2D imaging, in contrast to its 3D counterpart, is quite well developed and often readily available at high resolutions. Further, as described in Section 2, 2D scan images are sufficient for estimating stochastic descriptors such as one- and two-point correlation functions in addition to microstructural features. Such information may be employed to reconstruct 3D volumes [31]. One such approach, originally developed by Joshi [17] in two dimensions, and later extended by Quiblier to three dimensions, is the most widely used JQA approach [15]. In this approach, based on conditioning and truncation of Gaussian random fields, 3D binary image data are generated by simply using the one- and two-point autocorrelation functions computed from 2D images [18].

A slightly modified version of this approach was proposed in Bentz and Martys [28], and is employed in this work. In this approach, the first step consists of obtaining the porosity and two-point autocorrelation functions, as described in Section 2.1. Then, an initial three-dimensional image consisting of Gaussian distributed noise is generated using a uniform random number generator and the Box-Muller method to convert the uniform random deviates to normal deviates [28]. This 3D noise image, $N(x, y, z)$, is subsequently directly filtered (or convolved) with the normalized autocorrelation function, $F(x, y, z)$, defined as

$$
F(r)=F(x, y, z)=\frac{S_{2}\left(r=\sqrt{x^{2}+y^{2}+z^{2}}\right)-S_{2}(0)^{2}}{S_{2}(0)-S_{2}(0)^{2}}
$$

In this equation, $S_{2}(r)$ is the autocorrelation function defined in Eq. (4) and computed from the 2D image as described in Section 2.1. Also, the Cartesian pixel coordinates are converted to spherical coordinates for computing the value of the filter function, $F$ [28]. Once the filter coefficients are obtained, the resulting $3 \mathrm{D}$ image is then filtered as

$$
R(x, y, z)=\sum_{i=0}^{c} \sum_{j=0}^{c} \sum_{k=0}^{c} N(x+i, y+j, z+k) \times F(i, j, k)
$$

It may be noted that $c$ is the cutoff distance in pixels, and its value is chosen based on the observed correlation length, from the $S_{2}$ function. Typical values of $c$ are about 2-3 times the correlation length, and in this study, a cutoff value of $c=30$ was chosen for all cases, based on an observed correlation length of about 10 pixels. Also, typical reconstructed sample sizes consisted of 100 pixels on a side.

The resulting 3D image, $R$, is not binary-valued. Therefore, a thresholding operation is subsequently performed to binarize the generated 3D image. A histogram of all the values is constructed and the threshold value is obtained iteratively, so as to match the porosity of the porous material. It may also be noted that since this approach is based on a normalized autocorrelation function, any desired value of porosity may be chosen in the thresholding step [28]. In this study, we have employed porosity values as 
quoted by the manufacturer and described in [6], for thresholding operations; similar values are also employed for binarizing the starting multi-valued 2D image. However, for a few cases, the porosity values were varied to assess the effect of porosity on computed values of effective thermal conductivity, as discussed in Section 6.2.3.

The final operation step, as described by Bentz and Martys [28], involved correcting the reconstructed microstructures to match the hydraulic diameter computed from the 2D image. A surface-smoothing algorithm developed for modeling sintering [32] was employed to swap material and pore pixels on the surface. Further details on this approach may be found in [28]. In this work, we have not employed this algorithm. Instead, we use a 3D Gaussian smoothing operation, followed by a porosity-matching threshold. This approach was found to better match both the surface area (hydraulic diameter) as well as the correlation length, as described in Section 6.2. In the hydraulic radius-matching approach of [32], the highest curvature pixels are randomly selected and relocated to the lowest curvature regions, so as to match the hydraulic diameter by lowering the surface area. Such an approach is not a local smoothing approach, and leads to particle elongation, similar to sintering. In contrast, Gaussian smoothing is a local smoothing technique in which a filter matrix with different coefficients (weights) corresponding to differently spaced neighbors is generated based on the Gaussian distribution. The filter matrix is then employed to convolve the starting image, leading to local smoothing. The reconstruction procedure employed is summarized in Figure 2 for a 250-355 $\mu \mathrm{m}$ case. The 2D image from the starting 3D XMT stack (top left) is first binarized to match the manufacturer-specified porosity (top center). This binary image is then employed to construct the filter function, $F$, in Eq. (14) (top right). Subsequently, the resultant image, $R$ (bottom right), is obtained by convolving the 3D Gaussian noise image with a cutoff value of 30 pixels, as mentioned earlier. The image is smoothed via the 3D Gaussian smoothing operation and a representative $2 \mathrm{D}$ cross-section (bottom center) and 3D microstructure reconstruction (bottom right) are shown for the same $250-355 \mu \mathrm{m}$ case.

\subsubsection{Optimization-based reconstruction}

Other reconstruction techniques include optimization-based approaches such as that proposed by Yeong and Torquato [19], based on the simulated annealing technique. In this approach, the starting microstructure consists of a porosity-matching, random distribution of pore and material pixels. In each iteration of the algorithm, pixels are swapped so as to minimize an energy function, consisting of a weighted sum of any of the stochastic descriptors. Commonly employed functions include autocorrelation functions, lineal path functions and chord length distribution functions [19], [26]. The advantage of this approach is that it can produce microstructures with the desired level of agreement between the stochastic properties of the 3D microstructure and the base 2D images [19]. However, being 
iterative, the approach is typically computationally intensive and time-consuming. Furthermore, the cost of the approach increases rapidly as the number of descriptors increase, as the stochastic parameters must be computed after each pixel swap.

\section{Designed porous media}

The various properties of the two-point autocorrelation function, $S_{2}$, were described in Section 2.1. As may be observed from Figure $1 \mathrm{~b}$ ), the $S_{2}$ function for a porous material starts with a value of porosity $\Phi$ at the origin, and rapidly decreases to the asymptotic value of $\Phi^{2}$, and the distance after which the autocorrelation function becomes asymptotic is the correlation length. Pixels separated by a distance greater than this length are uncorrelated, and vice versa [19]. Based on these observations, Debye et al. [25] proposed a model consisting of an exponentially decaying function to represent porous media made up of random shapes and sizes:

$$
\frac{S_{2}(r)-\phi^{2}}{S_{2}(0)-\phi^{2}}=\exp (-r / a)
$$

in which $a$ is the correlation length defined earlier. Yeong and Torquato [19] generated various microstructures, termed Debye random media, based on this function and demonstrated its utility in generating physically realistic microstructures. However, it may be noted that the Debye model has a single parameter, the characteristic length. Further, being based on a simple exponential decay function, the model fails to account for the oscillations observed in the correlation functions computed for porous media consisting of round particles, such as the sintered beds of interest. In order to reproduce these damped oscillations in the present work, we propose the following model that is based on a spring-massdamper system analogy [33]:

$$
\frac{S_{2}(r)-\phi^{2}}{S_{2}(0)-\phi^{2}}=e^{-b c r}\left[\cos \left(b \sqrt{1-c^{2}}\right) r+\frac{d+b c}{b \sqrt{1-c^{2}}} \sin \left(b \sqrt{1-c^{2}}\right) r\right]
$$

It may be noted that this model satisfies the boundary conditions, Eq. (7). The model parameters, $b, c$ and $d$, control the correlation length, extent of oscillations (damping), and slope (hydraulic diameter) of the generated porous media.

We employ this model in conjunction with the $3 \mathrm{D}$ microstructure generation procedure described in the previous section, and demonstrate its utility in generating microstructures with tunable performance characteristics such as permeability and interfacial heat transfer coefficients, as described in Section 6.3. With the advent of additive manufacturing techniques such as 3D printing, user-defined high-performance wick structures with tailored fluid-thermal transport characteristics may be realizable in the near future. 


\section{Numerical modeling}

The microstructures generated are analyzed for their thermal-fluid properties using the computational procedure described in this section. The first step involves converting the generated 3D data (stack of 2D images) into viable computational meshes. Following this, the discretized version of the governing equations, continuity, momentum and energy, are solved in the 3D computational domain. The solution procedure is described only briefly here, and the readers are referred to our prior studies $([6,[30])$ for a detailed discussion.

\subsection{Mesh Generation}

Computational meshes are generated from the 3D image data employing the commercial image processing and meshing program Simpleware [34]. This tool has a variety of options for processing, and generating all tetrahedral, or mixed hexahedral and tetrahedral elements. In this work, we employ the 'ScanFE-Grid' algorithm and generate meshes with volumetric and boundary adaptation to optimize and reduce the number of cells in the mesh [6]. Further, a mix of hexahedral and tetrahedral elements is employed to further lower the overall mesh element count. The meshes so produced are then exported to the FLUENT solver [35], which is used for flow and heat transfer analysis. For computing effective thermal conductivity, both the material and pore spaces are considered, while flow and interfacial heat transfer analysis only require the pore space of the domain meshed. Figure 3 shows a sample solid and pore space domain, along with an inset of the generated computational mesh.

\subsection{Governing Equations and Boundary Conditions}

The continuity, momentum and energy equations for the steady, constant-property flow of an incompressible Newtonian fluid are given by [6]:

$$
\begin{gathered}
\frac{\partial}{\partial x_{i}}\left(\rho u_{i}\right)=0 \\
\frac{\partial}{\partial x_{j}}\left(\rho u_{j} u_{i}\right)=-\frac{\partial P}{\partial x_{i}}+\mu\left(\frac{\partial^{2} u_{i}}{\partial x_{j} x_{j}}\right) \\
\frac{\partial}{\partial x_{i}}\left(\rho C u_{i} T\right)=\frac{\partial}{\partial x_{i}}\left(k \frac{\partial T}{\partial x_{i}}\right)
\end{gathered}
$$

Two types of computational simulations are performed in the cuboidal computational domain shown in Figure 3: a) thermal conduction in the presence of an interstitial fluid, i.e., conduction through both the metal and pore regions, and b) permeability and convective transport through the pore space. In the former computations, a conjugate heat conduction problem is solved, with an imposed temperature 
gradient. In the latter, computations are performed only in the pore space, assuming a no-slip boundary condition on the metal-pore space interface. For the thermal conductivity computation, given temperatures are imposed on the two opposing faces of the cuboidal domain, while the lateral boundaries are assumed to be adiabatic; the heat conduction equation is solved in both the fluid and solid regions, and continuity of temperature and heat flux is imposed at the interface. For the flow problem, a pressure-outlet boundary condition is employed in conjunction with a velocity inlet boundary condition on opposite boundaries, while the lateral boundaries are assumed to be symmetry boundaries. Although a fully developed flow assumption is not made, we found that the cross-section averaged pressure achieved a linear profile within the sample for all the Reynolds numbers considered here. For computation of the Nusselt number, conduction through the metal is neglected and a constant heat flux boundary condition is imposed on the interface separating the pore space from the metal. As with the pressure drop computation, we do not explicitly impose a fully developed thermal condition; nevertheless, the Nusselt number was found to become constant in the flow direction for the sample sizes and flow conditions considered here.

\subsection{Solution Procedure}

For the domain sizes in this work, computational meshes of approximately 4 million cells are generated for computations involving only the pore space (permeability and interfacial heat transfer), while a mesh of approximately 7 million cells is used for computing effective thermal conductivity. The governing equations are solved using the commercial computational fluid dynamics (CFD) solver FLUENT [35], employing a second-order upwind scheme for the flow and heat transfer calculations. Pressure-velocity coupling is addressed using the SIMPLE algorithm, along with an algebraic multigrid algorithm for solving the linearized system of governing equations. Details of the numerical method may be found in [6]. Also, the governing equations are suitably under-relaxed to ensure proper convergence. The default convergence criterion in FLUENT, based on the scaled residuals, is employed for termination. For heat transfer computations, the flow equations are decoupled from the energy equation and hence the continuity and momentum equations are first solved to convergence. After this, the energy equation is solved to convergence, with the converged flow solution assumed fixed.

The following sections present the results obtained corresponding to microstructural characterization based on the autocorrelation function, along with fluid-thermal transport characteristics computed for the generated 3D microstructures.

\section{Results and discussion}

\subsection{Correlation-Based Transport}


We now present results for the microstructural and transport characteristics of the sintered wicks considered, as estimated via the autocorrelation function. Five training images are considered for each of the 45-75 $\mu \mathrm{m}, 106-150 \mu \mathrm{m}$, and 250-355 $\mu \mathrm{m}$ samples, as noted earlier. These images span the entire thickness of the scanned wicks from [6], and thereby provide a better representation of the random sintered wicks than a single scan.

\subsubsection{Autocorrelation function}

All the fifteen grayscale images of size $350 \times 350$ pixels, five each corresponding to the small, intermediate and large particle-size cases, are first thresholded and binarized. The porosity values as quoted by the manufacturer (Thermacore Inc.) and reported in [6] are used as inputs, and the threshold value is selected iteratively. Following this, the autocorrelation function is computed, as in Eq. (4) and Eq. (6). Figure 4 shows a starting grayscale image, the corresponding binarized image, and the computed autocorrelation function for the smallest particle-size case for all the five images considered. Also shown is an inset that highlights the behavior near the correlation length. The autocorrelation functions - the correlation length, slope near the origin, and the location and extent of the mimima and maxima computed from various cross-sections - are observed to be grouped closely together. This observation confirms that a $2 \mathrm{D}$ image is sufficient to describe a stochastic 3D system, and that the image may be obtained at any cross-section. In addition, the autocorrelation function rapidly decreases to the asymptotic value of $\Phi^{2}$ as described earlier.

Figure $1 \mathrm{~b}$ ) shows the autocorrelation functions for the three samples considered, computed for one 2D image per sample. The small and intermediate sized particle cases clearly demonstrate significant oscillations, with a distinct first minimum, indicating the presence of rounded particles of nearly uniform size [14]. On the other hand, the largest particle-size sample demonstrates little to no oscillations in the computed autocorrelation function, thereby indicating a non-uniformity of particle size in this sample. Similar observations were also reported in [14], in which the lack of oscillations in the computed autocorrelation function was attributed to the irregular particles present in the investigated sandstone microstructures.

\subsubsection{Microstructural characteristics}

The autocorrelation functions are also employed to compute microstructural characteristics, viz., average pore size and particle size, and surface area per unit volume, as noted in Section 2.2. The computed values may then be employed as inputs in Eq. (11) for estimating permeability. The average value and standard deviation computed from the five images considered are shown in Table 1. Also 
shown are fitted particle diameter values, which are usually employed in the absence of a well-defined particle size, and computed via the following equation

$$
D_{p, 3 D}=\frac{6(1-\phi)}{s}
$$

Table 1 shows that the particle sizes computed from Eq. (10) and Eq. (20) are in good agreement with each other, with a maximum percentage difference of only approximately $10 \%$.

The specific surface area, $s$, is computed from Eq. (8), employing the slope of $S_{2}$ at the origin. As the autocorrelation function is a rapidly decreasing function, and as resolutions below 1 pixel are not possible without actually increasing the original scan resolution, we employ a spline-fit from MATLAB [32] for fitting the data. The slope of the curve is then computed from the fitted data, and the mean and standard deviation values of the specific surface area reported (Table 1). Also shown are values calculated from the actual scans from our previous study [6]. There is excellent agreement overall - the differences observed for the smallest, intermediate and largest particle-size samples are only approximately $14 \%, 5 \%$ and $0.4 \%$, respectively, relative to the 3D microtomography data from [6].

Another microstructural characteristic of interest is the average pore size of the porous material, which features in correlations developed for predicting single- and two-phase heat transfer through these structures. In this study, we employ the definition of Dullien, Eq. (9), for computing the effective pore diameter, and the corresponding mean and standard deviation values may be found in Table 1. Also shown for comparison are the effective pore diameters computed from our past study [6] which employed the porosity and specific surface areas from 3D microtomography data. As indicated, percentage differences are approximately $11 \%, 5 \%$ and $1 \%$, respectively, for the smallest, intermediate and the largest sized samples, proving the utility of the correlation-based approach in providing a coarse estimate of capillary pressure of sintered beds.

The relatively larger differences observed for the smallest particle-size sample may be attributed to the scan resolution, i.e., number of pixels per particle in the original 2D image. Based on scan resolutions, and mean particle sizes from Table 1, it may be noted that we had roughly 20 pixels per particle for the largest and intermediate particle-size samples, while, we had only approximately 12 pixels per particle for the smallest particle-size sample. High-resolution scans with an improved number of pixels per particle are therefore desirable for improving the prediction of microstructural features via the two-point autocorrelation function.

As demonstrated in this study, the autocorrelation function may be employed to estimate important microstructural characteristics of random, irregular porous media such as the sintered particle wicks. Once estimated, the pore and particle sizes and the specific surface area may be employed in empirical/semi-empirical correlations for a coarse prediction of transport characteristics. Such 
approximate methods prove themselves particularly useful when analyzing a large dataset of samples, or when assessing new materials. For a refined analysis, however, 3D computational domains are needed. The prediction of permeability, interfacial heat transfer coefficient and effective thermal conductivity employing the JQA approach is discussed in the following section.

\subsection{Analysis of Transport in Reconstructed Porous Media}

\subsubsection{Porous medium reconstruction}

The modified JQA approach detailed in Section 3.2.1 is employed for generating 3D microstructures, based on three starting images for each of the large, intermediate and small particle-size samples. Figure 5 shows a starting 2D image, the unsmoothed 3D data generated along with a 2D slice through the crosssection, and the corresponding smoothed $3 \mathrm{D}$ data along with a $2 \mathrm{D}$ slice. The data shown correspond to a 250-355 $\mu \mathrm{m}$ sample. As indicated in Figure 5, the microstructure generated by the JQA approach is rough. Further, as may be noted from the slope of the autocorrelation function in Figure 6 a), it has a larger specific surface area relative to the starting microstructure. In [28], a post-processing operation was performed to match the hydraulic diameter of the reconstructed volume with that obtained via the starting 2D image, to correct this artifact. In this work, we employ an alternative approach based on Gaussian smoothing.

Gaussian smoothing is an image-processing technique used for blurring (smoothing) images so as to reduce the inherent noise. In this approach, the smoothing operation is carried out via the application of a Gaussian kernel to each pixel in the image. The Gaussian kernel requires the parameters sigma and filter size [36] for performing the smoothing, with larger values of sigma leading to increased blurring (smoothing). A sigma value of 2.0 was found to preserve both the slope and correlation distance for the various cases investigated here. The recommended value of $6 \times \operatorname{sigma}(=12)$ is employed for the filter size [36]. Figure 6 a) shows a comparison of the autocorrelation functions obtained from the starting $2 \mathrm{D}$ image, a slice from the generated unsmoothed 3D data, a slice from the smoothed 3D data smoothed via the hydraulic radius-matching procedure detailed in [28], and also a slice from the Gaussian smoothed 3D data. Although the autocorrelation function computed from the as-generated data preserves the correlation length and mean particle size well, it has a large slope at the origin. In contrast, the smoothing procedure from [28] is observed to preserve the slope of the autocorrelation function (surface area), but leads to an increase in correlation length and mean particle size, which may be attributed to the non-local smoothing nature of this approach, as discussed in Section 3.2.1. Instead, our approach is a local smoothing approach, and preserves both the slope as well as the mean particle size and correlation length, as indicated in Figure 6. In the light of these observations, we employ Gaussian smoothing for all cases investigated in this work. The smoothed data is then converted to a computational mesh for analyzing 
transport characteristics, viz., permeability, interfacial heat transfer coefficient and effective thermal conductivity. Figure 6 b) shows similarly computed autocorrelation functions, obtained from various cross-sectional images constituting a Gaussian smoothing-based reconstructed 3D stack for the 250-355 $\mu \mathrm{m}$ case.

\subsubsection{Permeability and interfacial Nusselt number}

The permeability of sintered beds is typically predicted via the Kozeny-Carman (K-C) correlation, Eq. (11). Computationally, permeability $K$ may be estimated by imposing velocity inlet-pressure outlet boundary conditions on either ends of the domain, and correlating the observed pressure drop to the superficial velocity $U_{\text {mod }}$ via the modified Darcy equation [6]:

$$
-\frac{\partial P}{\partial x_{i}}=\frac{\mu}{K} U_{\text {mod }}+\frac{\rho C_{E}}{\sqrt{K}} U_{\text {mod }}^{2}
$$

Table 2 shows permeability values predicted in this study, compared against those from the KozenyCarman correlation as well as permeability data from [6], in which x-ray microtomography was employed for reconstructing the 3D microstructure for numerically estimating fluid-thermal transport properties of sintered wicks. Predictions from the current work are in excellent agreement with the microtomography data, proving a successful reconstruction. This is expected, since we start with a matched porosity during reconstruction. Further, we also ensure that the slope of the autocorrelation function, as well as the correlation length, are matched with those from the starting 2D image, as shown in Figure 6 a). On the other hand, the Kozeny-Carmen correlation, developed for packed beds of unsintered particles, predicts the trends reasonably well, but significantly over-predicts the values themselves. Similar observations were also reported in [6], and the mismatch was attributed to non-spherical particles with rough interfaces. Table 2 also shows values of Ergun's coefficient $C_{E}$ computed in this study, along with those from [6]. As indicated, there are significant differences with respect to [6] in the computed Ergun's coefficient data, which may be attributed to the limited information contained in the two-point autocorrelation functions employed for reconstruction in this study. For higher Reynolds numbers, and better reconstruction of particulate media, alternative stochastic information such as the chord length distribution function and the lineal path function (e.g., [19], [26]) may be employed.

Interfacial heat transfer coefficient is computed for a constant heat flux case as in our earlier studies $([6,[30])$. A constant heat flux boundary condition is imposed at the solid-fluid interface, while the domain boundaries in the transverse direction are considered adiabatic. Since the flow field is decoupled from the energy equation, we begin the heat transfer computations with the converged flow-field solution, and once the energy equation is also converged, interfacial heat transfer coefficient and Nusselt number are computed as shown below: 


$$
h_{s f}=\frac{q^{\prime \prime}}{\left(\overline{T_{s f}}-\overline{T_{f}}\right)} ; N u_{s f}=\frac{h_{s f} D_{p}}{k}
$$

In this equation, $\overline{T_{s f}}$ is the average temperature of the solid-fluid interface, while $\overline{T_{f}}$ is the average fluid temperature in the pore region. Figure 7 shows a comparison of Nusselt number data computed from this work, along with those from [6] in which x-ray microtomography was employed to reconstruct the 3D geometry. Also shown are uncertainty bars from [6]. As may be noted, the data are in very good agreement with the past computations for low Re, falling within the observed uncertainties. For higher $\mathrm{Re}$, the data are seen to diverge from previous computations; for higher Re, reconstruction based on the S2 function may be unable to capture geometric characteristics adequately, and alternative stochastic functions such as the chord-length distribution function may need to be employed for improved prediction.

\subsubsection{Effective thermal conductivity}

For computing effective thermal conductivity in a direction $x_{i}$, a temperature gradient $\left(\frac{\partial T}{\partial x_{i}}\right)$ is imposed across the two ends of the cuboidal sample, holding the lateral faces adiabatic. No pressure gradient is imposed and fluid in the pore space is considered static. The effective thermal conductivity, $k_{\text {eff, }}$, is calculated for water-saturated cases, as:

$$
k_{e f f}=\frac{-\int \mathbf{J} \cdot d \mathbf{A}}{\left(\frac{\partial T}{\partial x_{i}}\right) A}
$$

In contrast to values of permeability and interfacial Nusselt number, effective thermal conductivity values of the reconstructed samples demonstrated a negligible influence of particle or pore size and depended mainly on the porosity. This may be attributed to the limited information contained in the twopoint autocorrelation function $S_{2}$ employed for reconstructions. The autocorrelation function does not seem to capture the information on inter-particle contact (neck) size, which is critical to thermal conduction in packed/sintered beds, as noted in [37]. To delineate this better, sample 2D microstructures from [6] are considered.

In [8], a sintering model was developed to predict microstructural evolution during sintering of randomly packed circular and spherical particles in two and three dimensions, respectively. The model was employed to quantify the influence of sintering neck size on thermal conductivity and permeability. Here, we took three snapshots from the 2D random spherical bed case from [8], as shown in Figure 8. 
Also shown are the corresponding normalized autocorrelation functions. As may be observed, sintering leads to improved inter-particle contact at the expense of decreasing surface area [8]. The corresponding autocorrelation function computed also indicates a decreasing slope. However, the growth of the sintering neck is not explicitly captured $-S_{2}$ simply captures the increase in particle size as sintering progresses. The 3D microstructures reconstructed via $S_{2}$ are also expected to follow a similar behavior. The limitations of $S_{2}$-based thermal conductivity predictions were also demonstrated in [38], in which other higher-order descriptors such as the chord length distribution function, and lineal path function were employed to improve thermal conductivity predictions. However, the improvement in thermal conductivity predictions was found to be small despite a significant increase in computational cost.

Furthermore, as the starting microstructure prior to filtering was a Gaussian noise image, it was observed that the effective thermal conductivity values were very similar to those obtained via the effective medium theory (EMT) model [37]:

$$
k_{e f f}=\frac{1}{4}\left((3 \phi-1) k_{2}+[3(1-\phi)-1] k_{1}+\sqrt{\left[(3 \phi-1) k_{2}+(3\{1-\phi\}-1) k_{1}\right]^{2}+8 k_{1} k_{2}}\right)
$$

Here, $k_{l}$ is the thermal conductivity of the surrounding matrix (water), while $k_{2}$ is the thermal conductivity of the dispersed phase (copper). This model assumes a random dispersion of the phases, and as shown in [37], provides a suitable bound for predicting effective thermal conductivity of porous materials. In our previous study we demonstrated that EMT model may be employed as an alternative to the recommended Maxwell models (e.g., [1]) for predicting effective thermal conductivity of sintered beds. The values predicted via the EMT model were within $\pm 50 \%$ of our detailed computations, in contrast to Maxwell models which resulted in differences on the order of $\pm 200 \%$.

Figure 9 shows a comparison of normalized thermal conductivity computed via the generated 3D microstructures and the EMT model, Eq. (24), for a range of porosity values. Also shown are the Maxwell models [1], and a few data points from the literature, Bodla et al. [6], and Januszwski et al. [39] obtained for sintered beds manufactured in the range of $60-70 \%$ porosity. The lower Maxwell model assumes bicontinuous behavior in the matrix phase, while the upper Maxwell model assumes bicontinuous behavior of the particulate phase. As may be observed, the $S_{2}$ reconstruction-based simulations and the EMT model are in excellent agreement with each other in the range of 0-60\% porosity. However, the EMT model has a percolation threshold of about $60 \%$, and the thermal conductivity value from this model begins to decreases rapidly around this value. Similarly, the lower Maxwell model decreases rapidly with porosity as indicated in Figure 9, and effective thermal conductivity values predicted via this model are lower than those obtained via the EMT model. On the other hand, our generated microstructures show bicontinuous behavior till about $80 \%$ porosity; consequently, our approach provides a higher value of effective thermal conductivity as compared to the EMT model in this range. However, our values are still 
considerably lower than the predictions via the upper Maxwell model, as the latter model assumes bicontinuous behavior of the particulate phase, indicating that the connectivity generated by our reconstruction lies in between that assumed by the two Maxwell models.

\subsection{Porous Media Design}

In addition to analyzing existing microstructures, the current methodology enables the development of new designed porous microstructures. The model for the autocorrelation function, Eq. (16), is employed and 3D data generated via the procedure detailed here.

Nine cases, three each with varying values for parameters $b, c$ and $d$ in Eq. (16), are analyzed for their influence on the generated microstructures. The starting values for these parameters are obtained by fitting the autocorrelation function for an intermediate particle-size sample case; the exact values considered are listed in Table 3. Also shown are values of coefficients for samples from the smallest and largest particle-size cases. A few snapshots of the generated dataset are shown as insets in Figure 10 and in Figure 11.

Figure 10 shows a comparison of autocorrelation functions for a few cases. Also plotted are corresponding autocorrelation functions computed from an unsmoothed and smoothed slice from the generated 3D data set. As indicated, parameter $b$ controls the correlation length and particle size of the porous material. Similarly, parameter $c$ controls the extent of damping in the oscillations, while parameter $d$ controls the specific surface area.

The employed reconstruction approach is found to predict the correlation functions of the damped cases well. However, for the case $c 1$ in Table 3, which corresponds to a significantly under-damped case, the approach fails to provide a useful reconstruction. This is again attributable to the limited information contained in the autocorrelation function. To further confirm this behavior, test cases from [40] and [14] corresponding to a sandstone microstructure, and a synthetically generated 3D spherical particle bed with overlapping particles, were considered. Figure 12 shows the starting image, along with three reconstructed slices. The sandstone microstructure, which has fewer oscillations (less rounded particles), is reconstructed quite well. However, for the latter case which has rounded particles which exhibit oscillatory behavior in the $S_{2}$ function, the clear distinction of individual particles (roundness) is lost in the reconstruction process. Similar observations were also reported in [38], in which a hybrid function incorporating the chord length distribution function and the autocorrelation function was proposed as a suitable alternative to employing the autocorrelation function alone. Nonetheless, commercial sintered wicks demonstrated fewer oscillations in the autocorrelation (Figure 1 and Figure 4), and this model may still be employed to obtain a successful reconstruction. Such designed porous materials with controlled features may eventually be manufactured via recently developed novel additive manufacturing processes 
such as 3D printing. Table 3 also shows computed values of permeability and Ergun's coefficient for the various cases investigated. As may be noted, an increase in the absolute value of parameters $b$ and $d$ typically lead to decreasing permeability, as they control correlation length and pore size, respectively, both of which affect permeability. On the other hand, permeability values were relatively invariant with respect to parameter $c$.

\section{Conclusions}

This study investigates the applicability of stochastic functions measured from two-dimensional images in predicting transport properties of random sintered beds. Individual 2D images from microtomography data corresponding to three commercial wick structures are employed for predicting their microstructural characteristics and transport properties. It is demonstrated that the two-point autocorrelation function may be readily used to predict pore size, particle size and specific surface area accurately. These characteristics appear in fluid-thermal transport property correlations developed for porous media such as the widely used Kozeny-Carman correlation, Eq. (11). Such a simple correlationbased approach may be employed to gain a first estimate of transport characteristics of new materials or for analyzing large datasets.

Furthermore, a 3D microstructure reconstruction procedure is developed and implemented for generating stochastically equivalent $3 \mathrm{D}$ beds based on a single $2 \mathrm{D}$ cross-section. Two-dimensional imaging, in contrast to its 3D counterpart, is well-developed. Hence, in the absence of readily available 3D scan data, the methodology developed here may be used as an alternative for predicting transport characteristics. The present computations are successfully compared against simulations performed on previous geometries constructed from 3D scans. Furthermore, the influence of porosity on random sintered beds is also investigated. It is shown that, compared to the effective medium theory, our random microstructures have a significantly improved percolation threshold (porosity at which material ceases to be bi-continuous), and hence demonstrated a higher value of effective thermal conductivity.

In addition to investigating existing random media, a novel model is proposed for controlling the microstructure of random wicks. Based on the characteristics of the autocorrelation function, and exploiting an analogy to a damped spring-mass system, a parameterized model similar to one available in the literature is proposed. The influence of various parameters in the model is investigated, and their physical relevance demonstrated. By combining optimization procedures with the proposed autocorrelation function, it is possible to optimally design wicks to achieve specific flow and heat transfer objectives. For example, wicks with desired capillary pressure may be designed by controlling parameter $b$ in the proposed model. Similarly, values of parameters $b$ and $d$ may be tuned to attain desired values of permeability. These user-designed wicks may be fabricated using advances in $3 \mathrm{D}$ printing and other 
additive manufacturing techniques, thereby radically improving the effectiveness of heat pipes and vapor chambers.

\section{Acknowledgements}

The authors acknowledge support for this work from industry members of the Cooling Technologies

Research Center, an NSF Industry/University Cooperative Research Center.

\section{References}

[1] A. Faghri, Heat pipe science and technology, Taylor \& Francis Washington, DC, 1995.

[2] P.D. Dunn, D.A. Reay, The heat pipe, Physics in Technology, 4 (1973) 187.

[3] S.V. Garimella, Y.K. Joshi, A. Bar-Cohen, R. Mahajan, K.C. Toh, V.P. Carey, M. Baelmans, J. Lohan, B. Sammakia, and F. Andros, Thermal challenges in next generation electronic systemssummary of panel presentations and discussions, IEEE Transactions on Components and Packaging Technologies, 25(4) (2003) 569-575.

[4] J.A. Weibel, S.V. Garimella, M.T. North, Characterization of evaporation and boiling from sintered powder wicks fed by capillary action, International Journal of Heat and Mass Transfer, 53 (2010) 4204-4215.

[5] F.A.L. Dullien, Porous media; fluid transport and pore structure, Academic press, San Diego, CA, 1979.

[6] K.K. Bodla, J.Y. Murthy, S.V. Garimella, Direct simulation of thermal transport through sintered wick microstructures, Journal of Heat Transfer, 134(1) (2012) 012602-012610.

[7] K.K. Bodla, J.Y. Murthy, S.V. Garimella, Evaporation analysis in sintered wick microstructures, International Journal of Heat and Mass Transfer, 61 (2013) 729-741.

[8] K.K. Bodla, J.A. Weibel, S.V. Garimella, Advances in fluid and thermal transport property analysis and design of sintered porous wick microstructures, Journal of Heat Transfer, 135 (2013) 061202061213.

[9] K.K. Bodla, J.Y. Murthy, S.V. Garimella, Resistance network-based thermal conductivity model for metal foams, Computational Materials Science, 50 (2010) 622-632.

[10]M. Van Geet, R. Swennen, M. Wevers, Towards 3-D petrography: application of microfocus computer tomography in geological science, Computers \& Geosciences, 27 (2001) 1091-1099.

[11]S.R. Stock, X-ray microtomography of materials, International Materials Reviews, 44 (1999) 141164.

[12] N. Kawase, M. Kato, H. Nishioka, H. Jinnai, Transmission electron microtomography without the “missing wedge" for quantitative structural analysis, Ultramicroscopy. 107 (2007) 8-15. 
[13]B. Van Meerbeek, M. Vargas, S. Inoue, Y. Yoshida, J. Perdigao, P. Lambrechts, G. Vanherle, Microscopy investigations. Techniques, results, limitations., American Journal of Dentistry, 13 (2000) 3D-18D.

[14] S.C. Blair, P.A. Berge, J.G. Berryman, Using two-point correlation functions to characterize microgeometry and estimate permeabilities of sandstones and porous glass, Journal of Geophysical Research: Solid Earth, 101 (1996) 20359-20375.

[15]A.P. Roberts, E.J. Garboczi, Elastic properties of a tungsten-silver composite by reconstruction and computation, Journal of the Mechanics and Physics of Solids, 47 (1999) 2029-2055.

[16] S. Adhikari, M.I. Friswell, Distributed parameter model updating using the Karhunen-Loève expansion, Mechanical Systems and Signal Processing. 24 (2010) 326-339.

[17]M. Joshi, A class of stochastic models for porous materials, Ph. D. thesis, University of Kansas, Lawrence, 1974.

[18]J.A. Quiblier, A new three-dimensional modeling technique for studying porous media, Journal of Colloid and Interface Science, 98 (1984) 84-102.

[19]C.L.Y. Yeong, S. Torquato, Reconstructing random media, Phys. Rev. E., 57 (1998) 495-506.

[20] A. Hajizadeh, A. Safekordi, F.A. Farhadpour, A multiple-point statistics algorithm for 3D pore space reconstruction from 2D images, Advances in Water Resources, 34 (2011) 1256-1267.

[21] Z. Jiang, W. Chen, C. Burkhart, Efficient 3D porous microstructure reconstruction via Gaussian random field and hybrid optimization, Journal of Microscopy. 252 (2013) 135-148.

[22] T. Zhang, Y. Du, Reconstructing Porous Media Using MPS, in: Multimedia and Signal Processing, Springer, 2012: pp. 341-348.

[23] D. Chen, Q. Teng, X. He, Z. Xu, Z. Li, Stable-phase method for hierarchical annealing in the reconstruction of porous media images, Physical Review E. 89 (2014) 013305.

[24]P. Debye, A.M. Bueche, Scattering by an inhomogeneous solid, Journal of Applied Physics, 20 (1949) 518-525.

[25]P. Debye, H.R. Anderson, H. Brumberger, Scattering by an inhomogeneous solid. II. The correlation function and its application, Journal of Applied Physics, 28 (1957) 679-683.

[26]S. Torquato, B. Lu, Chord-length distribution function for two-phase random media, Phys. Rev. E., 47 (1993) 2950-2953.

[27]J.G. Berryman, Measurement of spatial correlation functions using image processing techniques, Journal of Applied Physics, 57 (1985) 2374-2384.

[28]D.P. Bentz, N.S. Martys, Hydraulic radius and transport in reconstructed model three-dimensional porous media, Transport in Porous Media, 17 (1994) 221-238. 
[29]L.A. Giannuzzi, F.A. Stevie, A review of focused ion beam milling techniques for TEM specimen preparation, Micron. 30 (1999) 197-204.

[30] K.K. Bodla, J.Y. Murthy, S.V. Garimella, Microtomography-based simulation of transport through open-cell metal foams, Numerical Heat Transfer, Part A: Applications, 58 (2010) 527-544.

[31]H. Okabe, M.J. Blunt, Prediction of permeability for porous media reconstructed using multiple-point statistics, Phys. Rev. E., 70 (2004) 066135.

[32]D.P. Bentz, P.J.P. Pimienta, E.J. Garboczi, W.C. Carter, Cellular automaton simulations of surface mass transport due to curvature gradients: Simulations of sintering in 3-D., in: MRS Proceedings, 1991.

[33] S.S. Rao, Mechanical Vibrations, 4/E, Pearson Education India, 2003.

[34] ScanIP, ScanFE and ScanCAD tutorial guide for SIMPLEWARE 3.1, Simpleware Ltd., Exeter, UK, 2009.

[35] User's Guide for FLUENT 6.0, Fluent Inc., Lebanon, NH, 2002.

[36] MATLAB: The language of technical computing, version 6.5, The MathWorks, Inc. Natick, MA, 2002.

[37] J.K. Carson, S.J. Lovatt, D.J. Tanner, A.C. Cleland, Thermal conductivity bounds for isotropic, porous materials, International Journal of Heat and Mass Transfer, 48 (2005) 2150-2158.

[38] C.L.Y. Yeong, S. Torquato, Reconstructing random media. II. Three-dimensional media from twodimensional cuts, Phys. Rev. E., 58 (1998) 224-233.

[39] J. Januszewski, M. I. Khokar, A. S. Mujumdar, Thermal conductivity of some porous metals, Letters in Heat and Mass Transfer, 4 (2005) 417-423.

[40] P. Adler, J. Thovert, S. Bekri, F. Yousefian, Real porous media: Local geometry and transports, Journal of Engineering Mechanics, 128 (2002) 829-839. 


\section{List of Tables}

Table 1. Particle diameter, pore size and specific surface area from the present study, shown along with similar data from [6]. Mean values are shown, along with standard deviations in brackets.

Table 2. Comparison of permeability $\mathrm{K}$ and Ergun's coefficient $\mathrm{C}_{\mathrm{E}}$. Mean values are shown, along with standard deviations in brackets.

Table 3. Computed permeability and Ergun's coefficient data for various designed porous media. Also shown are fitted values of coefficients in Eq.(16), obtained for existing samples. 


\section{List of Figures}

Figure 1. a) Mesh for computing autocorrelation function. Pixelated grid is shown along with a few lattice points used for computation, and b) sample autocorrelation function computed for the three cases used in this study, shown along with the microtomography slices employed.

Figure 2. Typical workflow employed in this study for generating stochastically equivalent 3D microstructures from 2D images. .

Figure 3. a) Solid, and b) fluid (pore) domain with boundary conditions for the flow problem, shown for a reconstructed microstructure corresponding to the 250-355 $\mu \mathrm{m}$ case. Also shown is an inset with a zoomed-in view of mesh.

Figure 4.a) Original 2D microtomography image, b) corresponding thresholded and binarized image, and c) autocorrelation function computed from various starting images, shown for the 45-75 $\mu \mathrm{m}$ case. Also shown is a zoomed-in view around the correlation length, demonstrating oscillations in the autocorrelation function.

Figure 5. 3D reconstruction from thin sections - a) starting image, b) correlated, unsmoothed 3D image, and c) Gaussian smoothed 3D image, shown for the largest particle size. Insets in b) and c), demonstrate the effect of smoothing.

Figure 6. a) Comparison of autocorrelation functions computed from the original and resultant microstructures reconstructed using different methods, and b) comparison of autocorrelation functions computed via Gaussian smoothed reconstructed data, obtained employing various 2D cross-sections from the reconstructed $3 \mathrm{D}$ stack.

Figure 7. Prediction of Nusselt number as a function of modified Peclet number, compared against computations from [6].

Figure 8. Effect of sintering on normalized autocorrelation function, shown for the case of randomly packed circles from [8].

Figure 9. Effective thermal conductivity prediction, and comparison against literature.

Figure 10. Influence of parameters, ( a) $b$, (b) $c$, and (c) $d$ (Eq. (16)) on autocorrelation function, shown along with sample $2 \mathrm{D}$ images of generated microstructures.

Figure 11. Representative cross-sections from the reconstructed 3D stack, corresponding to different values for parameters, $b, c$, and $d$, respectively. Values for the parameters along with computed permeability values may be found in Table 3 .

Figure 12. Qualitative comparison of the developed reconstruction procedure shown for a) homogeneous sandstone from [40], and b) random hard spheres from [14]. 
Table 1. Particle diameter, pore size and specific surface area from the present study, shown along with similar data from [6]. Mean values are shown, along with standard deviations in brackets.

\begin{tabular}{|c|c|c|c|c|c|c|}
\hline Sample & $D_{p}(\mu m)$ & $\begin{array}{c}D_{p, 3 D} \\
(\mu \mathrm{m})[6]\end{array}$ & $D_{H}(\mu m)$ & $\begin{array}{c}D_{H, 3 D} \\
(\mu \mathrm{m})[6]\end{array}$ & $s\left(\mathbf{m}^{-1}\right)$ & $\begin{array}{c}\text { s from } \\
\text { XMT } \\
\left(\mathrm{m}^{-1}\right)[6]\end{array}$ \\
\hline $\begin{array}{c}45-75 \\
\mu \mathrm{m}\end{array}$ & $\begin{array}{l}67.69 \\
(0.0)\end{array}$ & 74.3 & $\begin{array}{l}69.69 \\
(1.50)\end{array}$ & 62.64 & $\begin{array}{c}38953.2 \\
(1229.66)\end{array}$ & 35281.31 \\
\hline $\begin{array}{c}106-150 \\
\mu \mathrm{m}\end{array}$ & $\begin{array}{l}111.69 \\
(3.78)\end{array}$ & 102 & $\begin{array}{c}114.66 \\
(4.36)\end{array}$ & 120.80 & $\begin{array}{c}24188 \\
(2859.72)\end{array}$ & 21192.09 \\
\hline $\begin{array}{c}250-355 \\
\mu \mathrm{m}\end{array}$ & $\begin{array}{l}193.6 \\
(9.84)\end{array}$ & 215 & $\begin{array}{c}239.4 \\
(11.39)\end{array}$ & 236.47 & $\begin{array}{l}10894.2 \\
(486.64)\end{array}$ & 10656.02 \\
\hline
\end{tabular}


Table 2. Comparison of permeability K and Ergun's coefficient $\mathrm{C}_{\mathrm{E}}$. Mean values are shown, along with standard deviations in brackets.

\begin{tabular}{cccccc}
\hline Sample & $\begin{array}{c}\text { Permeability } \\
\left(\mathbf{m}^{2}\right)\end{array}$ & $\begin{array}{c}\text { Permeability } \\
\text { from XMT } \\
\left(\mathbf{m}^{2}\right)[\mathbf{6}]\end{array}$ & $\begin{array}{c}\text { Permeability from } \\
\text { Kozeny-Carman, } \\
\text { Eq. }(\mathbf{1 1})\left(\mathbf{m}^{2}\right)\end{array}$ & $\mathbf{C}_{\mathbf{E}}$ & C $_{\mathbf{E}}$ from [6] \\
\hline $45-75$ & $2.67 \times 10^{-11}$ & $2.51 \times 10^{-11}$ & $6.44 \times 10^{-11}$ & 0.381 & \\
$\mu \mathrm{m}$ & $\left(8.1 \times 10^{-13}\right)$ & $\left(4.30 \times 10^{-13}\right)$ & & $\left(8.58 \times 10^{-4}\right)$ & 0.266 \\
$106-150$ & $5.11 \times 10^{-11}$ & $7.89 \times 10^{-11}$ & $3.46 \times 10^{-10}$ & 0.379 & 0.267 \\
$\mu \mathrm{m}$ & $\left(1.39 \times 10^{-11}\right)$ & $\left(1.54 \times 10^{-11}\right)$ & & $\left(2.99 \times 10^{-2}\right)$ & \\
$250-355$ & $2.68 \times 10^{-10}$ & $2.63 \times 10^{-10}$ & & 0.440 & 0.249 \\
$\mu \mathrm{m}$ & $\left(9.11 \times 10^{-11}\right)$ & $\left(5.15 \times 10^{-11}\right)$ & & $\left(6.55 \times 10^{-2}\right)$ & \\
\hline
\end{tabular}


Table 3. Computed permeability and Ergun's coefficient data for various designed porous media. Also shown are fitted values of coefficients in Eq.(16), obtained for existing samples.

\begin{tabular}{cccccc}
\hline Sample/case & B & $\mathbf{c}$ & $\mathbf{d}$ & $\begin{array}{c}\text { Permeability } \\
\left(\mathbf{m}^{2}\right)\end{array}$ & $\mathbf{C}_{\mathbf{E}}$ \\
\hline $45-75 \mu \mathrm{m}$ & 0.458 & 0.7 & -0.215 & - & - \\
$106-150 \mu \mathrm{m}$ & 0.278 & 0.735 & -0.139 & - & - \\
$250-355 \mu \mathrm{m}$ & 0.076 & 0.854 & -0.085 & - & - \\
b1 & 0.2 & 0.735 & -0.139 & $4.37 \times 10^{-11}$ & 0.448 \\
b2 & 0.4 & 0.735 & -0.139 & $2.71 \times 10^{-11}$ & 0.434 \\
b3 & 0.6 & 0.735 & -0.139 & $2.70 \times 10^{-11}$ & 0.380 \\
c1 & 0.278 & 0.3 & -0.139 & $4.70 \times 10^{-11}$ & 0.525 \\
c2 & 0.278 & 0.6 & -0.139 & $5.43 \times 10^{-11}$ & 0.443 \\
c3 & 0.278 & 0.9 & -0.139 & $5.64 \times 10^{-11}$ & 0.456 \\
d1 & 0.278 & 0.735 & -0.05 & $5.53 \times 10^{-11}$ & 0.400 \\
d2 & 0.278 & 0.735 & -0.2 & $4.47 \times 10^{-11}$ & 0.446 \\
d3 & 0.278 & 0.735 & -0.4 & $2.42 \times 10^{-11}$ & 0.502 \\
\hline
\end{tabular}




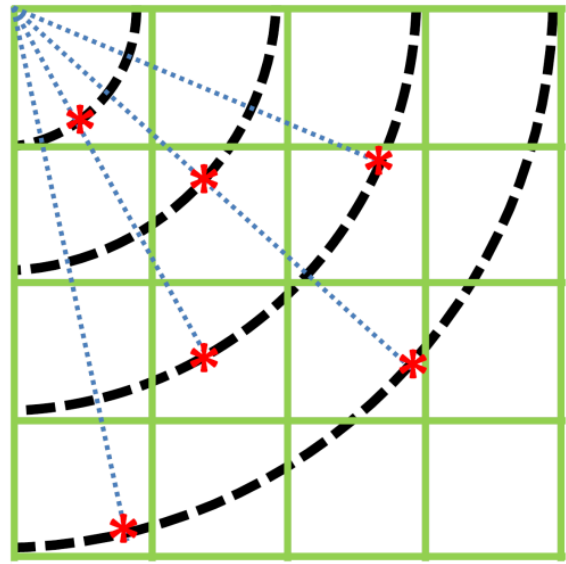

a)

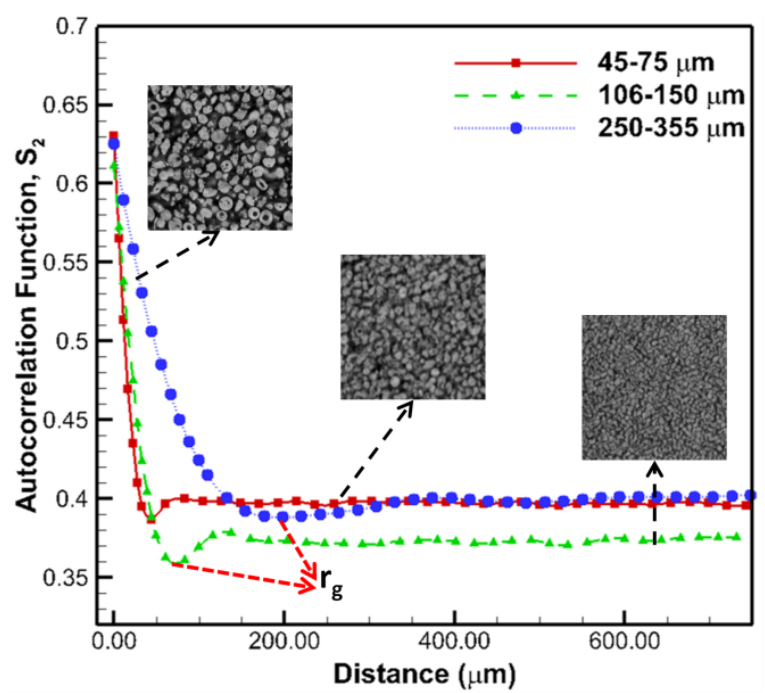

b)

Figure 1. a) Mesh for computing autocorrelation function. Pixelated grid is shown along with a few lattice points used for computation, and b) sample autocorrelation function computed for the three cases used in this study, shown along with the microtomography slices employed. 


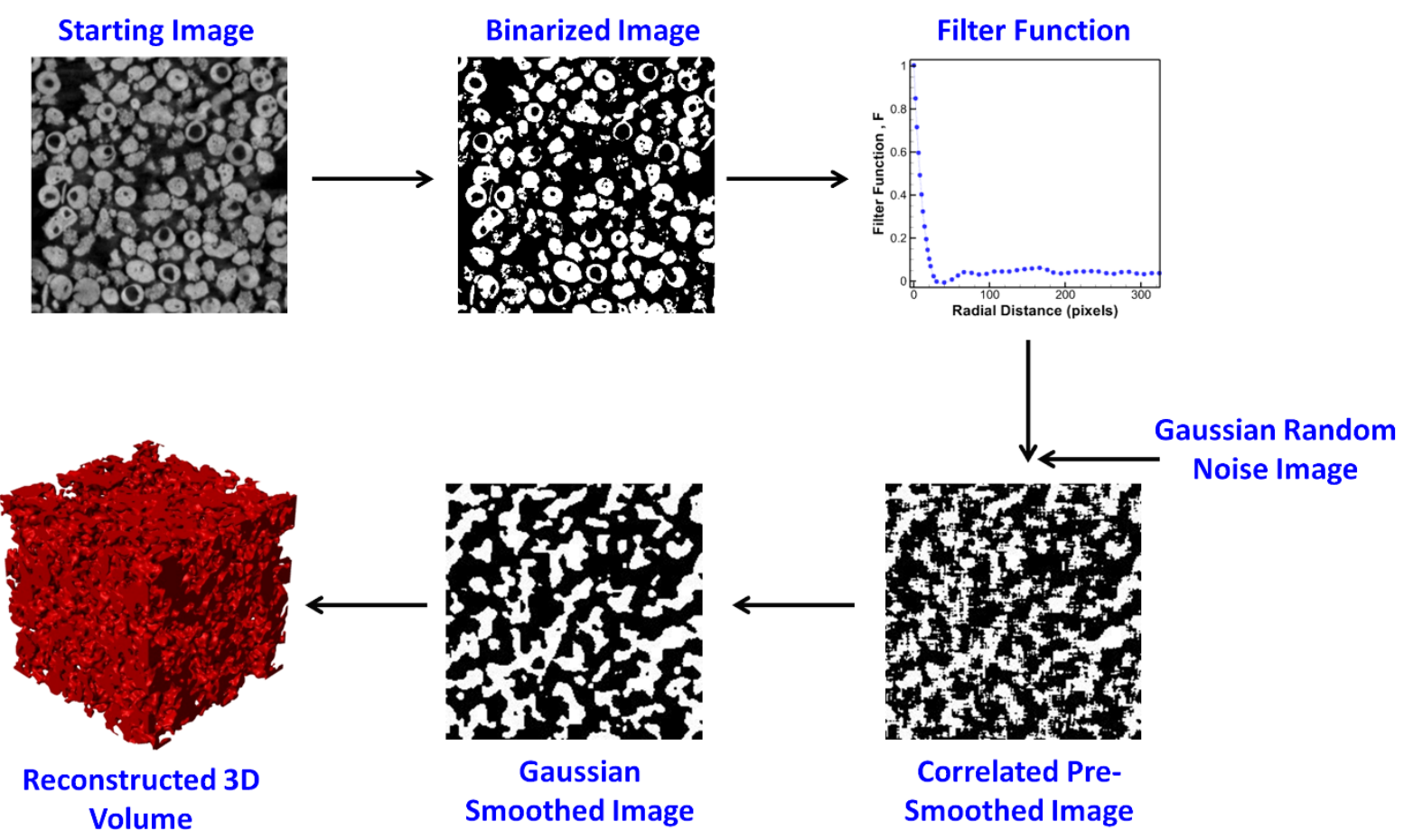

Figure 2. Typical workflow employed in this study for generating stochastically equivalent 3D microstructures from 2D images. . 


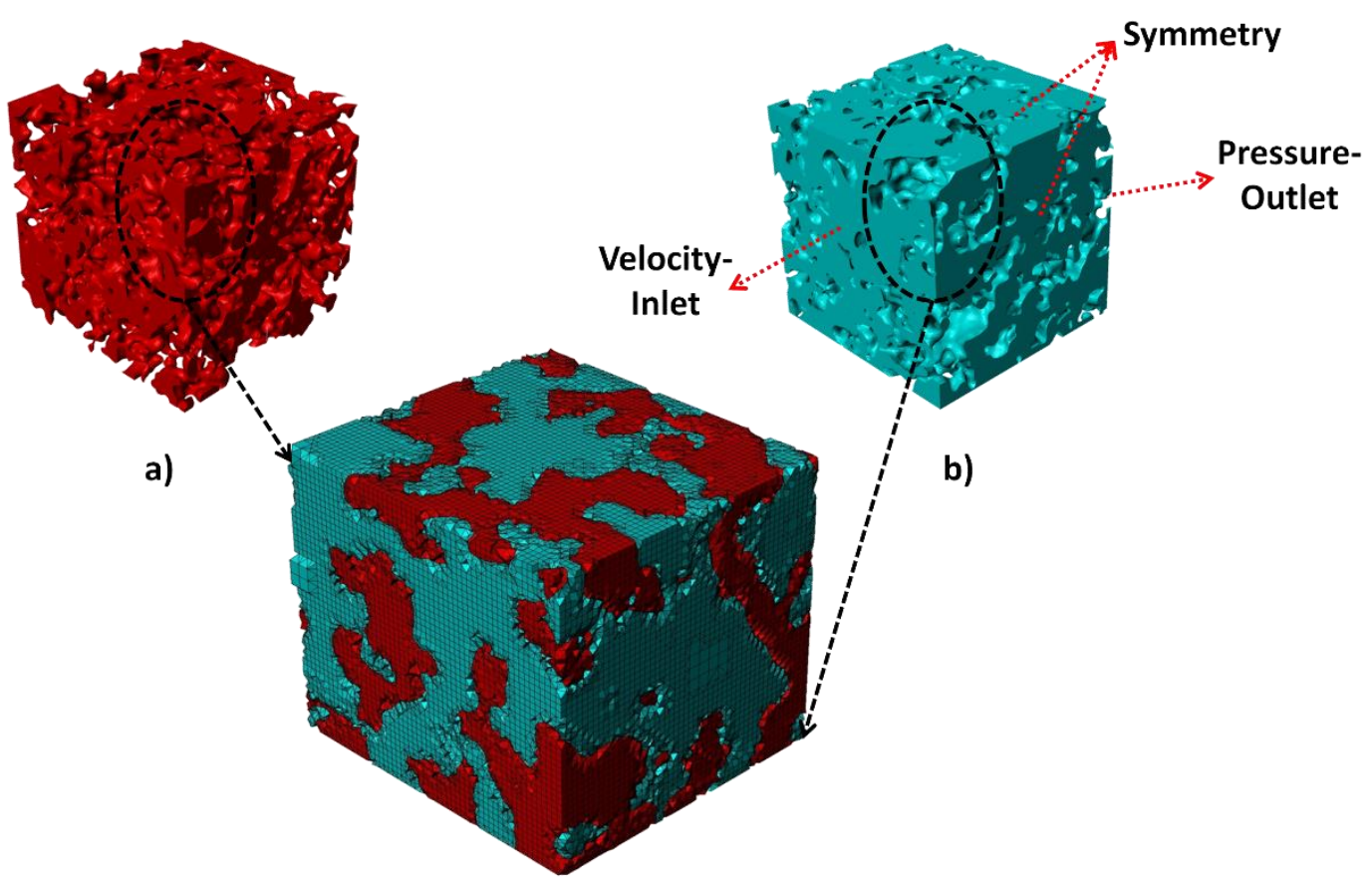

Figure 3. a) Solid, and b) fluid (pore) domain with boundary conditions for the flow problem, shown for a reconstructed microstructure corresponding to the 250-355 $\mu \mathrm{m}$ case. Also shown is an inset with a zoomed-in view of mesh. 


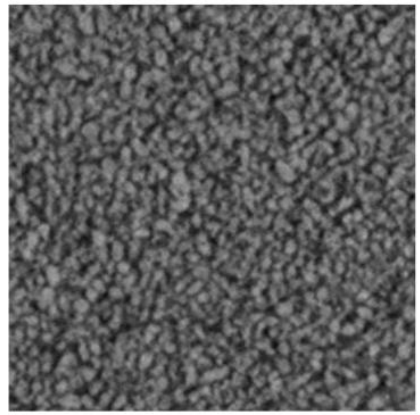

a)

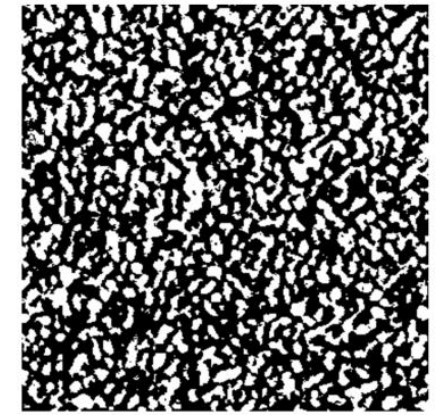

b)

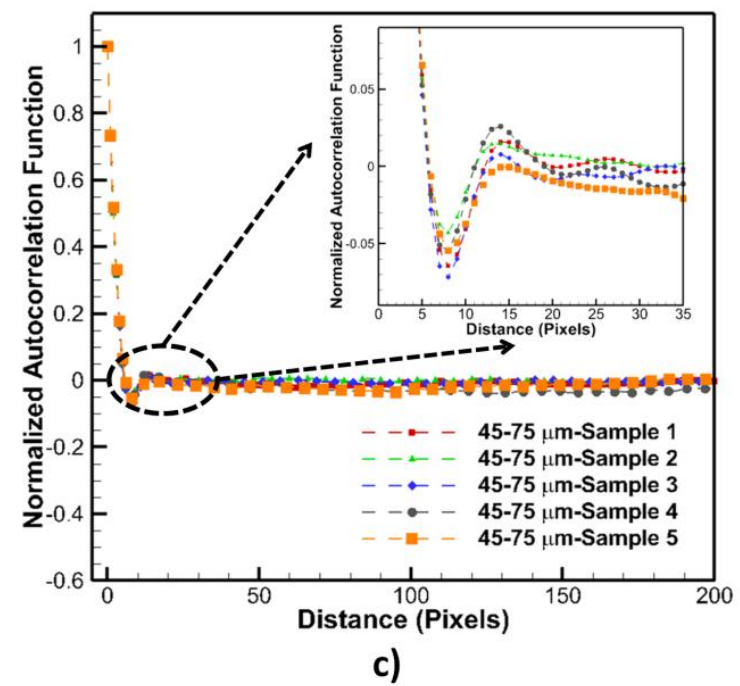

Figure 4.a) Original 2D microtomography image, b) corresponding thresholded and binarized image, and c) autocorrelation function computed from various starting images, shown for the 45-75 $\mu \mathrm{m}$ case. Also shown is a zoomed-in view around the correlation length, demonstrating oscillations in the autocorrelation function. 


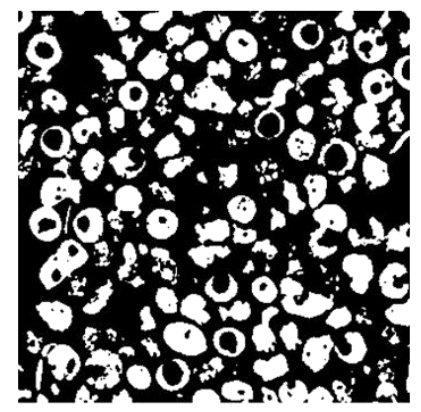

a)
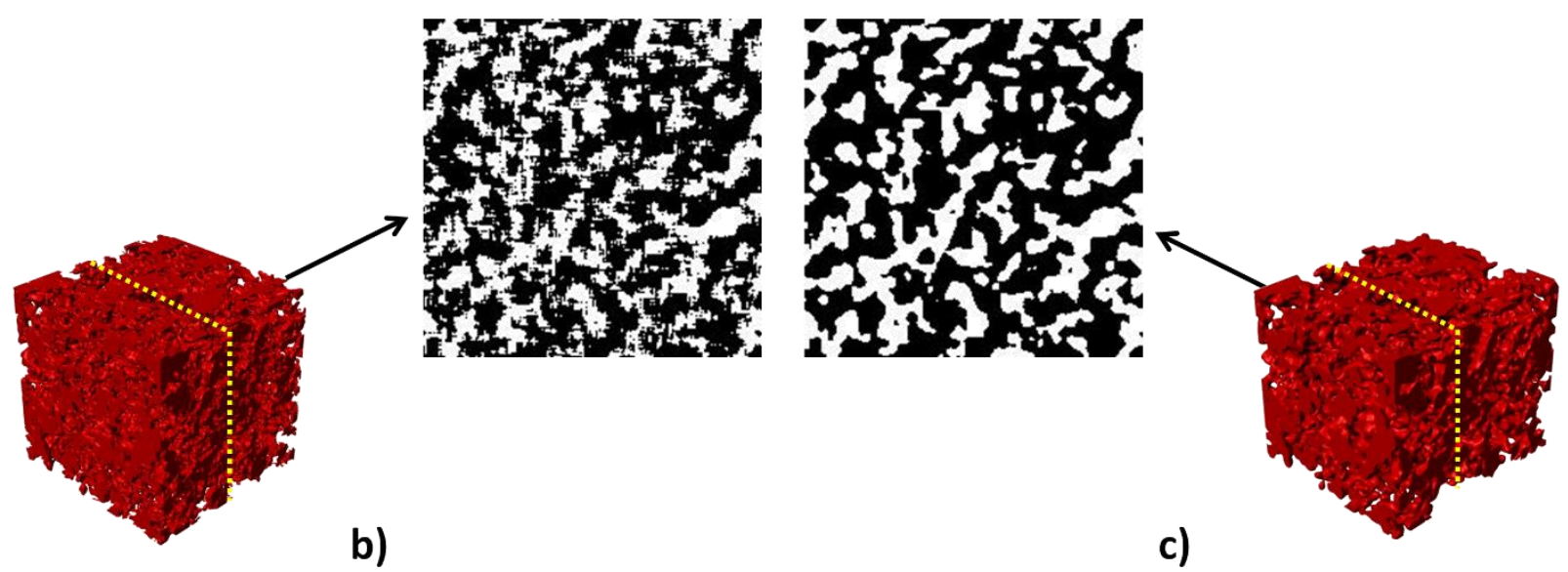

Figure 5. 3D reconstruction from thin sections - a) starting image, b) correlated, unsmoothed 3D image, and c) Gaussian smoothed 3D image, shown for the largest particle size. Insets in b) and c), demonstrate the effect of smoothing. 


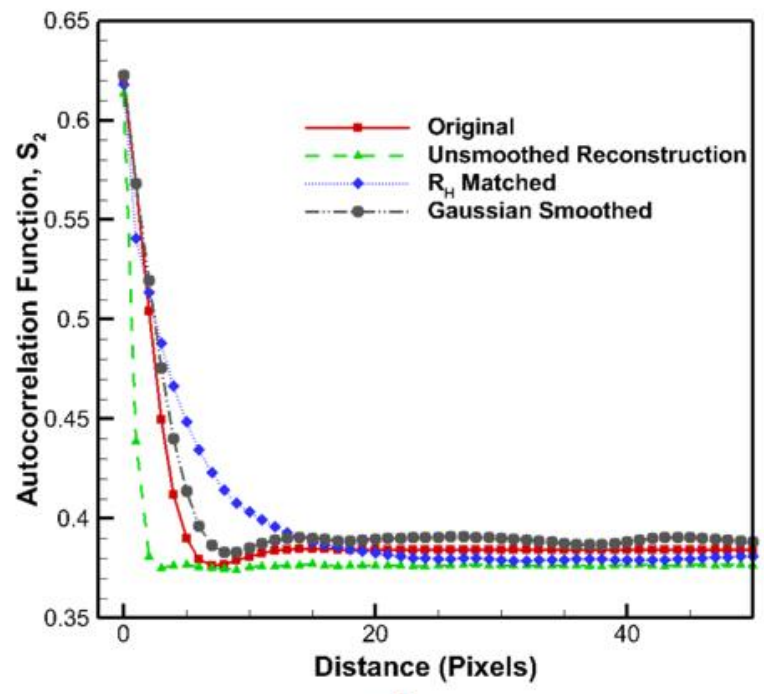

a)

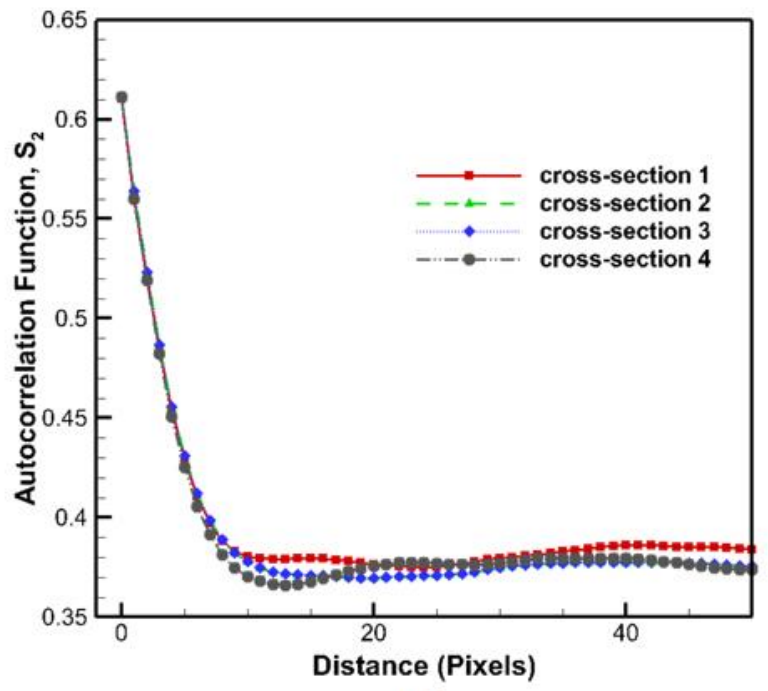

b)

Figure 6. a) Comparison of autocorrelation functions computed from the original and resultant microstructures reconstructed using different methods, and b) variation of autocorrelation functions computed via Gaussian smoothed reconstructed data for multiple 2D cross-sections within the reconstructed 3D stack. 


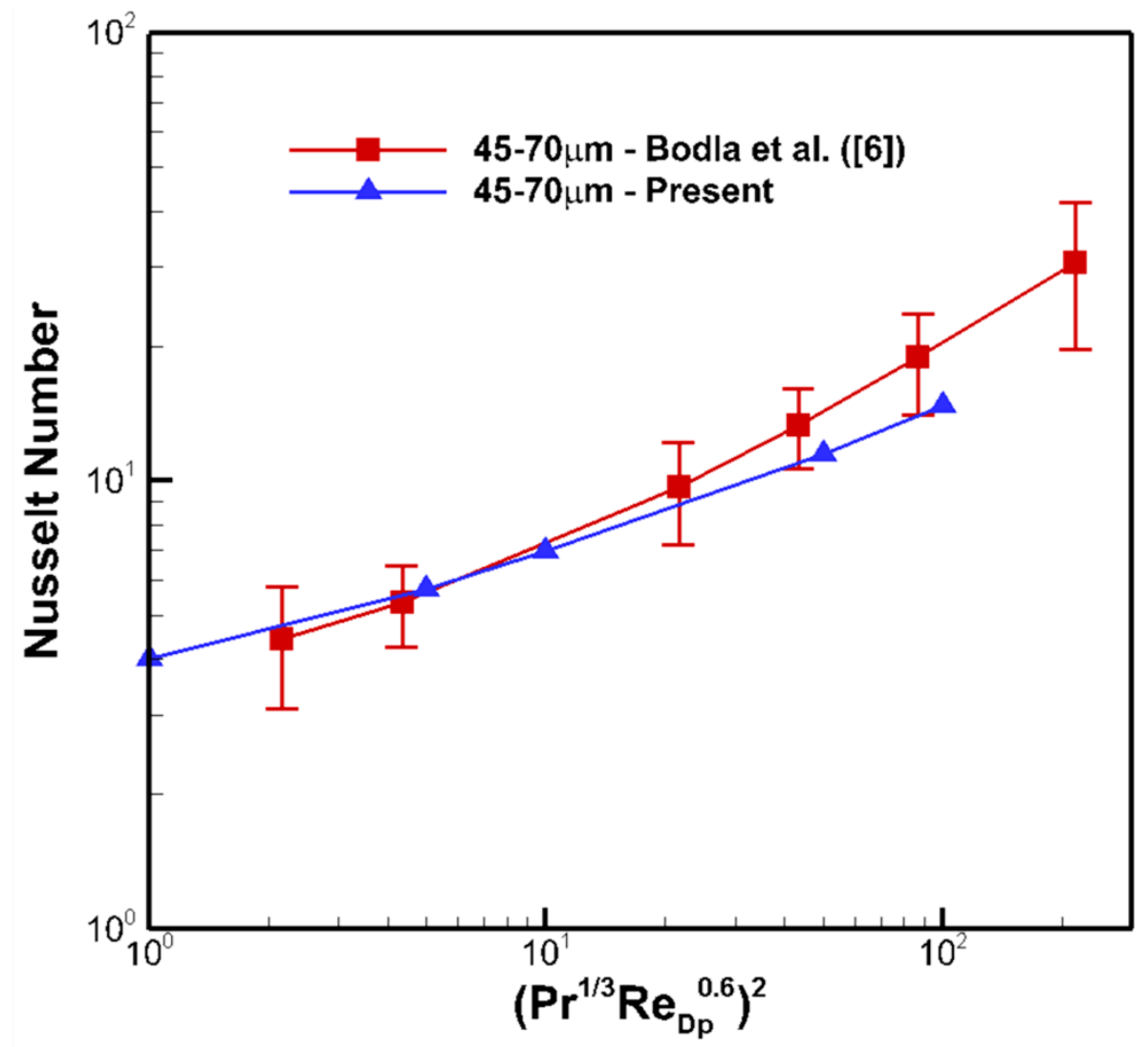

Figure 7. Prediction of Nusselt number as a function of modified Peclet number, compared against computations from [6]. 


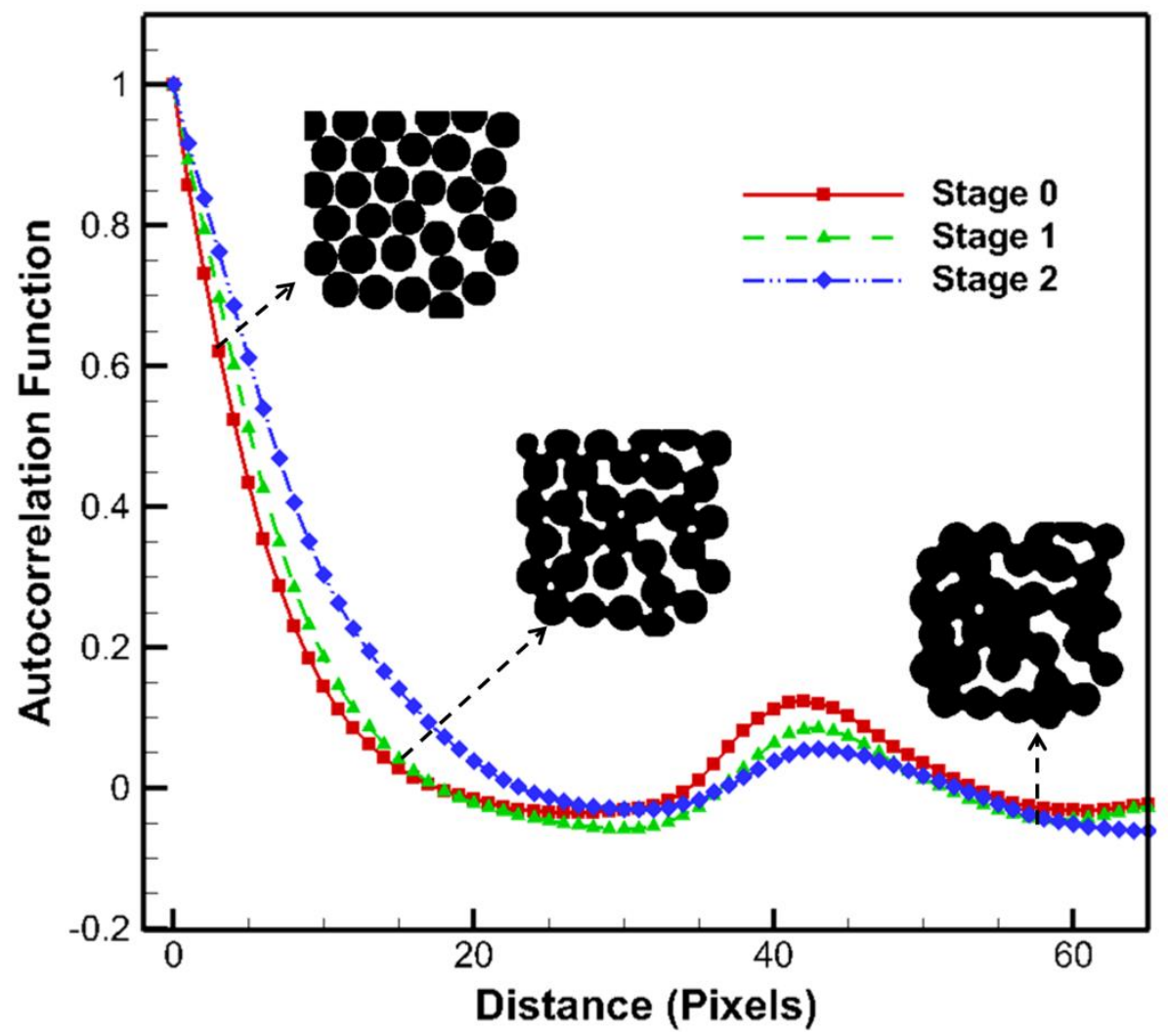

Figure 8. Effect of sintering on normalized autocorrelation function, shown for the case of randomly packed circles from [8]. 


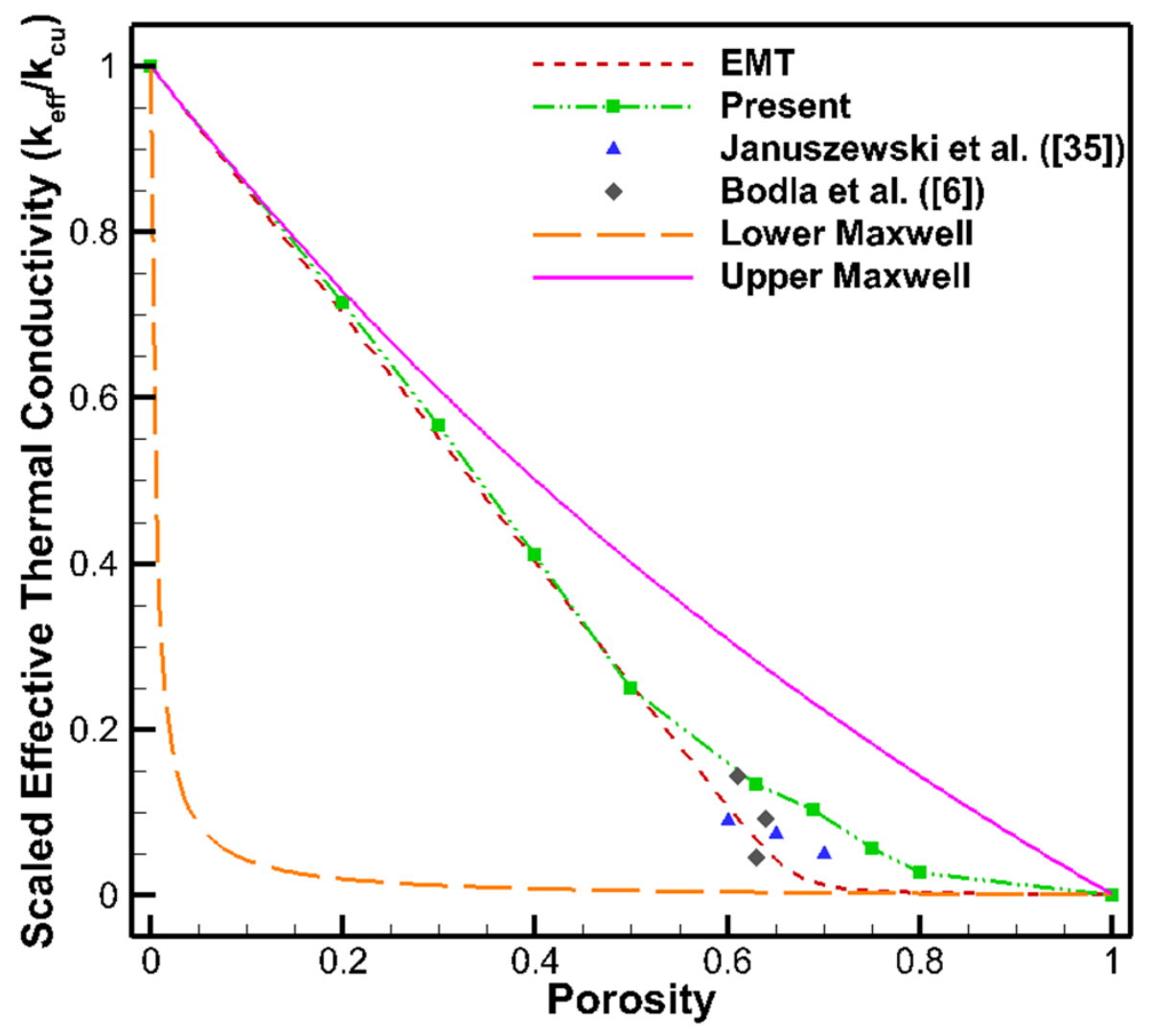

Figure 9. Effective thermal conductivity prediction, and comparison against literature. 


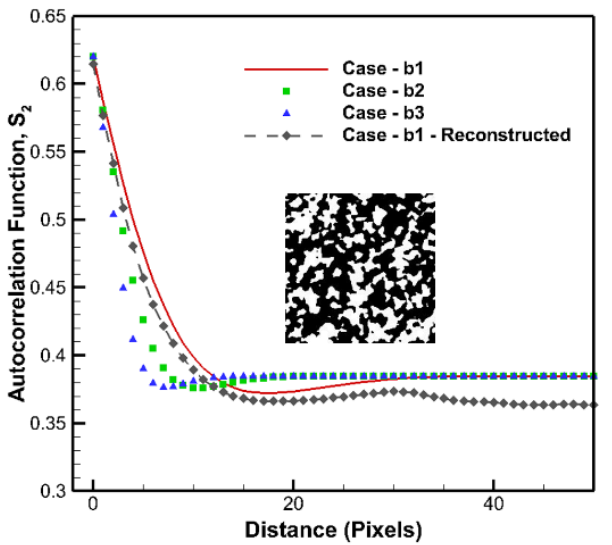

a)

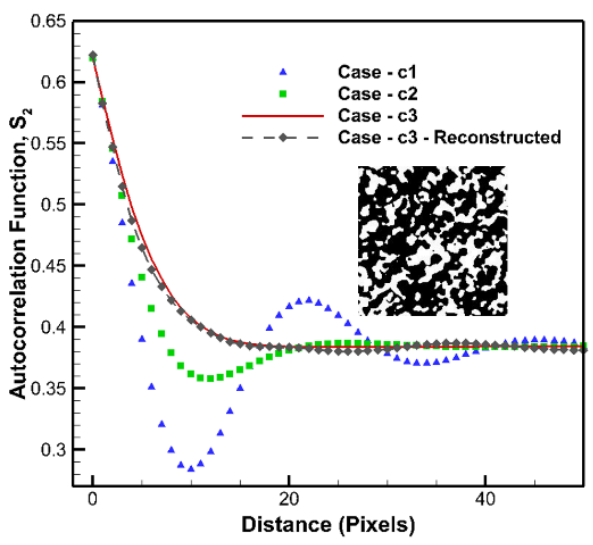

b)

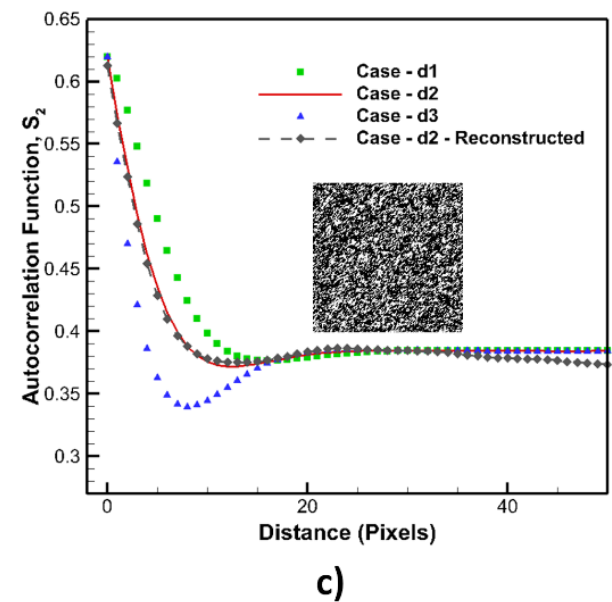

Figure 10. Influence of parameters, ( a) $b$, (b) $c$, and (c) $d$ (Eq. (16)) on autocorrelation function, shown along with sample 2D images of generated microstructures. 


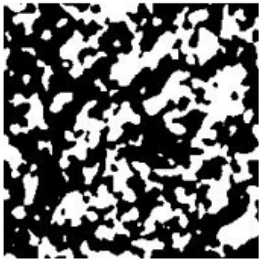

b1

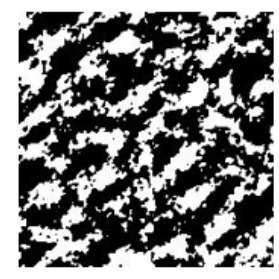

c1

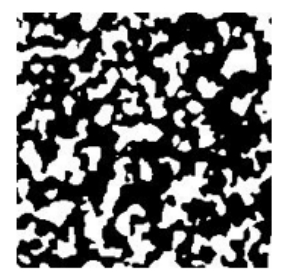

d1

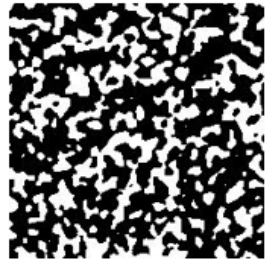

b2

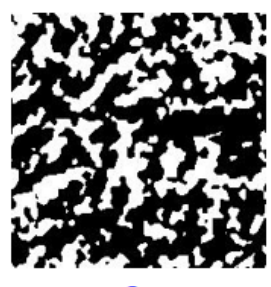

c2

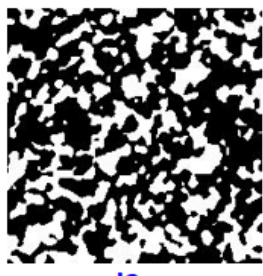

d2

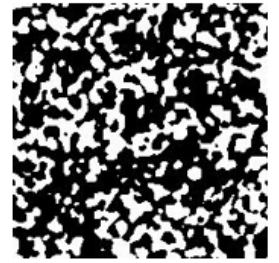

b3
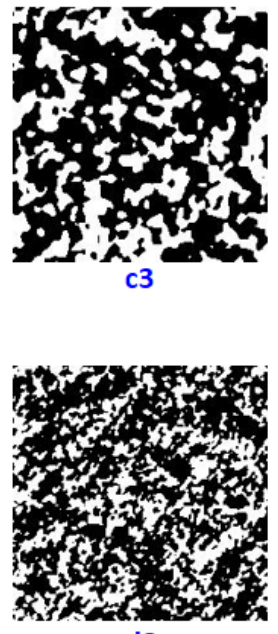

d3

Figure 11. Representative cross-sections from the reconstructed 3D stack, corresponding to different values for parameters, $b, c$, and $d$, respectively. Values for the parameters along with computed permeability values may be found in Table 3 . 

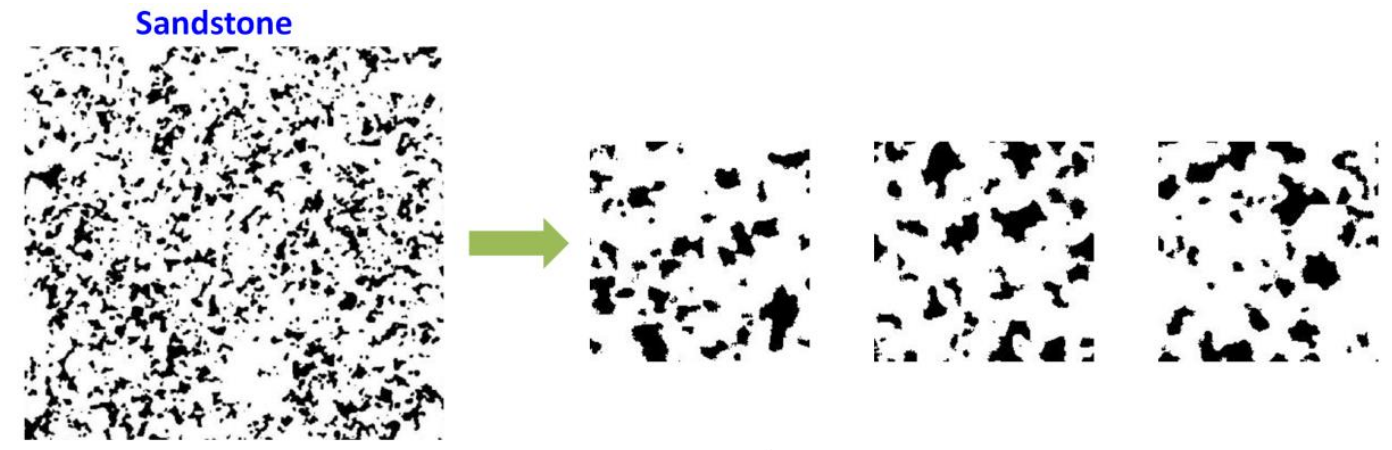

a)
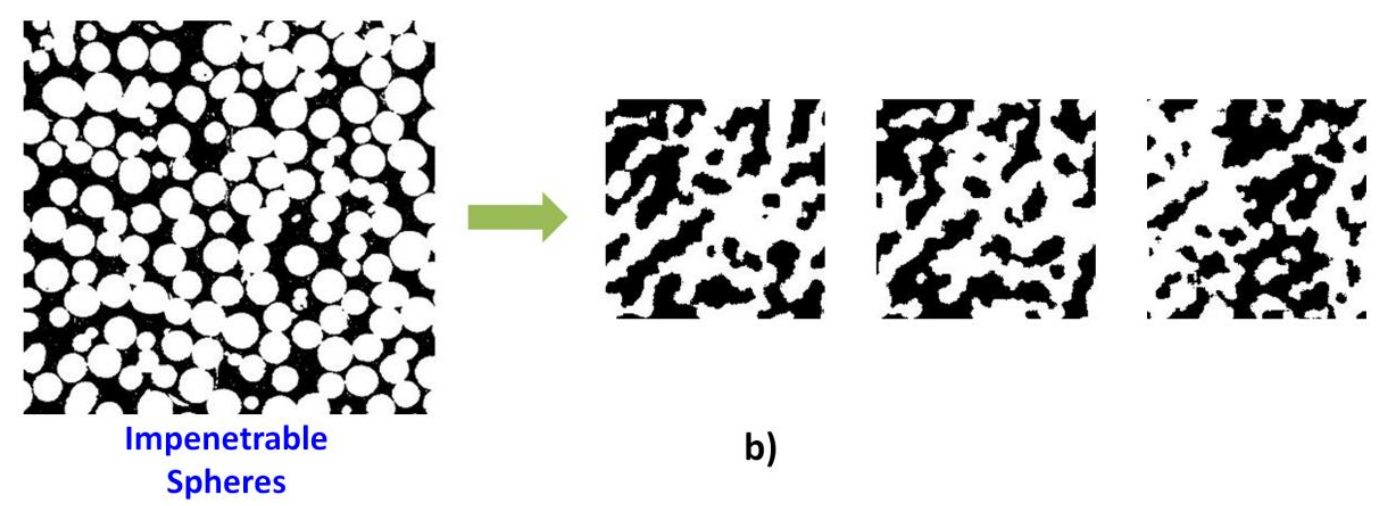

b)

Figure 12. Qualitative comparison of the developed reconstruction procedure shown for a) homogeneous sandstone from [40], and b) random hard spheres from [14]. 